\title{
Spectroscopic mapping of the planetary nebula NGC $6302^{\star}$
}

\author{
A. B. Rauber ${ }^{1}$, M. V. F. Copetti ${ }^{1}$, and A. C. Krabbe $^{2}$ \\ ${ }^{1}$ Laboratório de Análise Numérica e Astrofísica, Departamento de Matemática, Universidade Federal de Santa Maria, \\ Av. Roraima 1000, CEP 97119-900 Santa Maria, RS, Brazil \\ e-mail: alinerauber@gmail.com \\ 2 Universidade do Vale do Paraíba, Av. Shishima Hifumi 2911, CEP 12244-000 São José dos Campos, SP, Brazil
}

Received 31 December 2013 / Accepted 17 January 2014

\section{ABSTRACT}

\begin{abstract}
Context. Most spectroscopic studies of planetary nebulae (PNe) are based on data from specific parts of the objects. Considering the high complexity of their morphological structures, integrated or average values across an observed region can only provide limited information of their ionic, density, and temperature structures.

Aims. We investigate the spatial variation of the physical and chemical properties of NGC 6302, a high-ionization PNe, with a complex bipolar structure.

Methods. Spatially resolved long-slit spectroscopic data were obtained from 11 parallel and equally spaced directions over the nebula. Maps of emission line fluxes, line ratios, electron temperature $T_{\mathrm{e}}$, electron density $N_{\mathrm{e}}$, and ionic abundances $\left(\mathrm{He}^{+}, \mathrm{He}^{2+}, \mathrm{N}^{0}, \mathrm{~N}^{+}, \mathrm{O}^{0}\right.$, $\mathrm{O}^{+}, \mathrm{O}^{2+}, \mathrm{S}^{+}, \mathrm{S}^{2+}, \mathrm{Ne}^{2+}, \mathrm{Ar}^{2+}, \mathrm{Ar}^{3+}$, and $\mathrm{Ar}^{4+}$ relative to $\mathrm{H}^{+}$) were constructed with a spatial resolution of $1.45^{\prime \prime} \times 1^{\prime \prime}$ for a large portion of the nebula over an area of $11^{\prime \prime} \times 250^{\prime \prime}$. Electron densities were estimated from the [S II] $\lambda 6716 / \lambda 6731$ and [Ar IV] $\lambda 4711 / \lambda 4740$ line ratios, and electron temperatures were estimated from the $[\mathrm{O}$ III $](\lambda 4959+\lambda 5007) / \lambda 4363$ and $[\mathrm{N} \mathrm{II}](\lambda 6548+\lambda 6584) / \lambda 5755$ ratios. Integrated spectra were also obtained and 142 lines were identified.

Results. The electron density maps show a peaked distribution, with the densest area at the circumstellar region, reaching $N_{\mathrm{e}} \approx$ $40000 \mathrm{~cm}^{-3}$, and decreasing to $N_{\mathrm{e}} \leq 1000 \mathrm{~cm}^{-3}$ at the lobes. Knots were observed in the $N_{\mathrm{e}}$ (S II) map, with $2000 \mathrm{~cm}^{-3}<N_{\mathrm{e}}<$ $3000 \mathrm{~cm}^{-3}$. The $N_{\mathrm{e}}$ (Ar IV) map suggests the presence of an inner region with $N_{\mathrm{e}} \approx 20000 \mathrm{~cm}^{-3}$ that extends from the center through the east lobe. A homogeneous temperature distribution was found for both $T_{\mathrm{e}}(\mathrm{O}$ III $)$ and $T_{\mathrm{e}}(\mathrm{N}$ II $)$. Small temperature fluctuations on the plane of the sky of $t_{\mathrm{s}}^{2}(\mathrm{~N}$ II $)=0.0071$ and $t_{\mathrm{s}}^{2}(\mathrm{O}$ III $)=0.0043$ were estimated.

Conclusions. In general, the total abundances obtained are similar to the maximum found in the ionic abundance maps of the dominant ionic species. This demonstrates the potential of spatially resolved abundance studies for establishing lower limits of the total abundances based on ionic abundances, for checking the ionization correlation factors, and in some cases, for determining the total abundances directly from maps of ionic abundances.
\end{abstract}

Key words. planetary nebulae: individual: NGC 6302 - ISM: abundances

\section{Introduction}

The bipolar planetary nebula (PN) NGC 6302 has been classified as Type I (Peimbert \& Torres-Peimbert 1983; Kingsburgh \& Barlow 1994) with high helium and nitrogen abundances. Narrow band images in different emission lines obtained with the Hubble Space Telescope show the conspicuous bipolar morphology and the complex small-scale structures of the nebula (see Matsuura et al. 2005, Fig. 7). The lobes and the innermost parts of the nebula are expanding with a velocity increasing with the radial distance (Szyszka et al. 2011). At the center, there is a high extinction molecular torus, oriented north-south, roughly perpendicular to the axis of the lobes, and expanding slowly with a velocity of $8 \mathrm{~km} \mathrm{~s}^{-1}$ (Peretto et al. 2007). Within it, there is an ionized torus whose nature and evolution is not completely understood.

Because of the high extinction of its circumstellar region, the central star of NGC 6302 has only recently been identified at the position $\alpha=17^{\mathrm{h}} 13^{\mathrm{m}} 44.39, \delta=-37^{\circ} 06^{\prime} 12^{\prime \prime} \cdot 93(\mathrm{~J} 2000)$ (Szyszka et al. 2009). It is among the hottest central stars of PNe with an effective temperature higher than $250000 \mathrm{~K}$, as indicated by

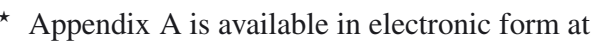
http://www. aanda.org
}

the detection of emission lines from very high-ionization species (Ashley \& Hyland 1988; Casassus et al. 2000; Feibelman 2001).

In the literature, there are a number of studies of the physical conditions of NGC 6302 based on spectroscopic observations in the optical, ultraviolet, and mid- and near-infrared ranges (Aller et al. 1981; Pottasch \& Beintema 1999; Casassus et al. 2000; Groves et al. 2002; Tsamis et al. 2003; Krabbe \& Copetti 2005). Different regions of the nebula were observed with different telescopes, also using different slit configurations and aperture sizes. Although these works give a reliable overview of the object, they do not provide detailed information of different morphological structures. Wright et al. (2011) presented a 3D photoionization model of NGC 6302 based on data compiled from these studies. The best-fitting model was obtained using a homogeneous elemental abundance distribution and three principal components for the density: a very dense circumstellar disk, a pair of lobes with a constant density, and a higher density component at the inner region of the lobes. Knots were also introduced to simulate density inhomogeneities. Most of the emission line ratios in the literature were reproduced, but some could not be matched. Significant differences were found between some model ionic abundances and those reported by Tsamis et al. (2003).

Krabbe \& Copetti (2005) published a spatially resolved spectroscopic study of NGC 6302. However, they only studied the 
spatial variation in the physical conditions along a single projected line on this nebula, based on long-slit spectroscopic data obtained at one slit position. Richer 2D spatially resolved data can be obtained by using integral field units or from multiple parallel long-slit spectroscopic observations. The derived 2D maps of line ratios and physical properties provide better constraints for the 3D modeling of photoionized nebulae. However, only a small number of PNe have been studied using a spectroscopic mapping technique. Some examples are NGC 6369 (Monteiro et al. 2004), NGC 40 (Leal-Ferreira et al. 2011), NGC 5882 (Tsamis et al. 2008), NGC 6153 (Tsamis et al. 2008), and NGC 7009 (Tsamis et al. 2008; Phillips et al. 2010).

This paper reports the results of a spectroscopic mapping of the PN NCG 6302. Two-dimensional spectral maps and spatial profiles of emission line fluxes, relative line intensities, and physical properties, such as electron density, electron temperature, and ionic and total abundances, were produced with a spatial resolution of approximately $1.45^{\prime \prime} \times 1^{\prime \prime}$. In Sect. 2 , we describe the observations and the data reduction procedure. The results are presented and discussed in Sect. 3, and a summary of our conclusions is presented in Sect. 4.

\section{Observations and data reduction}

Long-slit spectra of NGC 6302 were obtained on 29, 30, and 31 August 2008 with the Goodman spectrograph at the $4.1 \mathrm{~m}$ Southern Astrophysical Research Telescope (SOAR), Cerro Pachón, Chile. A $4096 \times 4096$ pixels Fairchild CCD was used. The spatial scale was approximately $0.15^{\prime \prime} \mathrm{pixel}^{-1}$. The slit was $3.7^{\prime}$ long and $1^{\prime \prime}$ wide. The $3001 \mathrm{~mm}^{-1}$ and the $6001 \mathrm{~mm}^{-1}$ gratings were used. The $3001 \mathrm{~mm}^{-1}$ spectra covered the range from $3600 \AA$ to $9150 \AA$, with a reciprocal dispersion of $1.3 \AA$ pixel $^{-1}$ and a spectral resolution of $8.9 \AA$, and the $6001 \mathrm{~mm}^{-1}$ spectra from $3550 \AA$ to $6300 \AA$, with $0.65 \AA$ pixel $^{-1}$ and resolution of $4.5 \AA$. The slit was oriented east-west and centered at 11 different equally spaced declinations. The declination offsets relative to the adopted reference star 2MASS J17134915-3706075 $\left(\alpha=17^{\mathrm{h}} 13^{\mathrm{m}} 49^{\mathrm{s}}, \delta=-37^{\circ} 06^{\prime} 08^{\prime \prime}, \mathrm{J} 2000\right)$ ranged from $6^{\prime \prime} \mathrm{S}$ to $4^{\prime \prime} \mathrm{N}$, with steps of $1^{\prime \prime}$. The exposure times at each slit position were $3 \times 300 \mathrm{~s}$ for the $6001 \mathrm{~mm}^{-1}$ grating and $3 \times 250 \mathrm{~s}$ for the $3001 \mathrm{~mm}^{-1}$ grating. Figure 1 shows the slit positions on an $R$-band image of NGC 6302 from the ESO survey, obtained with the Aladin software made available by the Centre de Données astronomiques de Strasbourg.

The spectra were reduced using the IRAF ${ }^{1}$ standard procedures for bias subtraction, flat-fielding, and cosmic-ray rejection. From each 2D spectrum we extracted a series of 150 1D spectra from contiguous sectors with $1.45^{\prime \prime}$ (10 pixels) length along the slit axis using the apall task. On each 2D spectrum, we identified and measured the positions along the slit of the detected field star to assure the extraction of the 1D spectra from the same bands in different exposures and slit positions. After adding together the 1D spectra from different exposures, 1650 1D spectra were obtained for each grating. Spectra of an $\mathrm{Hg}$-Ar lamp were taken for the wavelength calibration, but in some parts of the spectra, their emission lines were too sparse or weak. Thus, the wavelength calibration was refined using the main emission lines observed in the spectra of NGC 6302 itself. Spectra of the standard stars LTT 9491 and LTT 7379 were obtained for flux calibration.

\footnotetext{
1 IRAF is distributed by the National Optical Astronomy Observatory (NOAO), which is operated by the Association of Universities for Research in Astronomy (AURA), Inc., under cooperative agreement with the National Science Foundation.
}

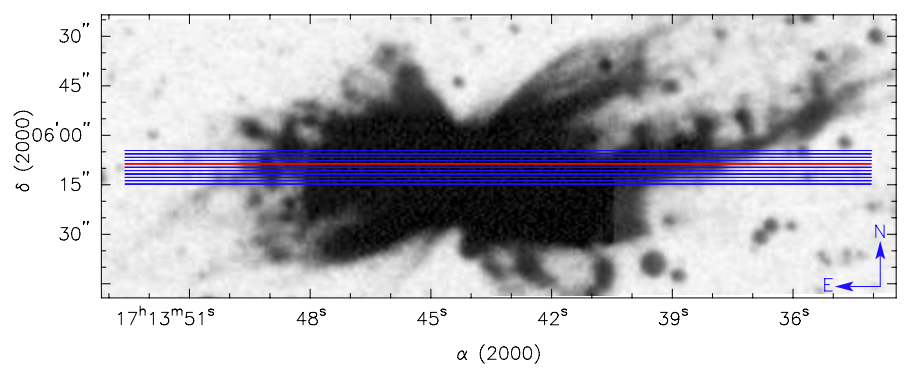

Fig. 1. Positions of the slit on an R-band image of NGC 6302 from the ESO survey, obtained with the ALADIN software made available by the Centre de Données astronomiques de Strasbourg. The slit positions are represented by the red and blue lines. The red line indicates the offset $0^{\prime \prime}$

Table 1. Emission lines selected for the maps.

\begin{tabular}{|c|c|c|c|}
\hline$\lambda(\AA)$ & Ion & Terms & $J_{\text {lower }}-J_{\text {upper }}$ \\
\hline $3726.03+$ & [O II $]$ & ${ }^{4} S^{o}-{ }^{2} D^{o}$ & $3 / 2-3 / 2$ \\
\hline 3728.82 & {$[\mathrm{O} \mathrm{II}]$} & ${ }^{4} S^{o}-{ }^{2} D^{o}$ & $3 / 2-5 / 2$ \\
\hline 3869.07 & [Ne III] & ${ }^{3} \mathrm{P}-{ }^{1} \mathrm{D}$ & $2-2$ \\
\hline 4340.47 & H I & ${ }^{2} \mathrm{P}^{\mathrm{o}}-{ }^{2} \mathrm{D}$ & $1 / 2-3 / 2-1 / 2-9 / 2$ \\
\hline 4363.21 & [O III $]$ & ${ }^{1} \mathrm{D}-{ }^{1} \mathrm{~S}$ & $2-0$ \\
\hline 4471.51 & He I & ${ }^{3} \mathrm{P}^{\mathrm{o}}-{ }^{3} \mathrm{D}$ & $0-2-1-3$ \\
\hline 4685.71 & He II & $3-4$ & $1 / 2-5 / 2-1 / 2-7 / 2$ \\
\hline $4711.37+$ & [Ar IV] & ${ }^{4} S^{o}-{ }^{2} D^{o}$ & $3 / 2-5 / 2$ \\
\hline 4713.22 & He I & ${ }^{3} \mathrm{P}^{\mathrm{o}}-{ }^{3} \mathrm{~S}$ & $0-2-1$ \\
\hline 4740.17 & [Ar IV] & ${ }^{4} S^{o}-{ }^{2} D^{o}$ & $3 / 2-3 / 2$ \\
\hline 4861.33 & H I & ${ }^{2} \mathrm{P}^{\mathrm{o}}-{ }^{2} \mathrm{D}$ & $1 / 2-3 / 2-1 / 2-7 / 2$ \\
\hline 4958.91 & [O III $]$ & ${ }^{3} \mathrm{P}-{ }^{1} \mathrm{D}$ & $1-2$ \\
\hline 5006.84 & [O III] & ${ }^{3} \mathrm{P}-{ }^{1} \mathrm{D}$ & $2-2$ \\
\hline 5200.26 & {$[\mathrm{~N} \mathrm{I}]$} & ${ }^{4} \mathrm{~S}^{\mathrm{o}}-{ }^{2} \mathrm{D}^{\mathrm{o}}$ & $3 / 2-5 / 2$ \\
\hline 5754.59 & {$[\mathrm{~N}$ II $]$} & ${ }^{1} \mathrm{D}-{ }^{1} \mathrm{~S}$ & $2-0$ \\
\hline 5875.67 & He I & ${ }^{3} \mathrm{P}^{\mathrm{o}}-{ }^{3} \mathrm{D}$ & $0-2-1-3$ \\
\hline 6300.30 & {$[\mathrm{O} \mathrm{I}]$} & ${ }^{3} \mathrm{P}-{ }^{1} \mathrm{D}$ & $2-2$ \\
\hline 6312.06 & [S III $]$ & ${ }^{1} \mathrm{D}-{ }^{1} \mathrm{~S}$ & $2-0$ \\
\hline $6548.05+$ & {$[\mathrm{N}$ II $]$} & ${ }^{3} \mathrm{P}-{ }^{1} \mathrm{D}$ & $1-2$ \\
\hline $6562.85+$ & H I & ${ }^{2} \mathrm{P}^{\mathrm{o}}-{ }^{2} \mathrm{D}$ & $1 / 2-3 / 2-1 / 2-5 / 2$ \\
\hline 6583.45 & {$[\mathrm{~N}$ II $]$} & ${ }^{3} \mathrm{P}-{ }^{1} \mathrm{D}$ & $2-2$ \\
\hline $6716.44+$ & [S II $]$ & ${ }^{4} S^{o}-{ }^{2} D^{o}$ & $3 / 2-5 / 2$ \\
\hline 6730.82 & {$[\mathrm{~S} \mathrm{II}]$} & ${ }^{4} S^{o}-{ }^{2} D^{o}$ & $3 / 2-3 / 2$ \\
\hline 7005.40 & [Ar V] & ${ }^{3} \mathrm{P}-{ }^{1} \mathrm{D}$ & $2-2$ \\
\hline 7135.80 & [Ar III] & ${ }^{3} \mathrm{P}-{ }^{1} \mathrm{D}$ & $2-2$ \\
\hline
\end{tabular}

The line fluxes were obtained from a single or multiple Gaussian profiles fitting between two given limits and over a local continuum. These measurements were made with the splot routine of the IRAF package. The error associated with the line flux was estimated by $\sigma_{\text {flux }}^{2}=\sigma_{\text {cont }}^{2}+\sigma_{\text {line }}^{2}$, where $\sigma_{\text {cont }}$ and $\sigma_{\text {line }}$ are the errors due to the continuum baseline determination and the Poisson error of the line, respectively.

\section{Results}

Emission-line flux maps of NGC 6302 with a spatial resolution of $1.45^{\prime \prime} \times 1^{\prime \prime}$ were constructed for each of the lines listed in Table 1. These are the main recombination lines of H I, He I, and He II lines, as well as the collisional excitation lines (CELs) used to compute the physical conditions in the nebula. To avoid spurious measurements of any specific line at a position where it was too weak and to define the boundaries of the nebula in different emission lines, we discarded the measurements in the pixels where the line had an intensity peak below 2.5 times the 
A. B. Rauber et al.: Spectroscopic mapping of the planetary nebula NGC 6302

$\log F(\lambda 3727)$
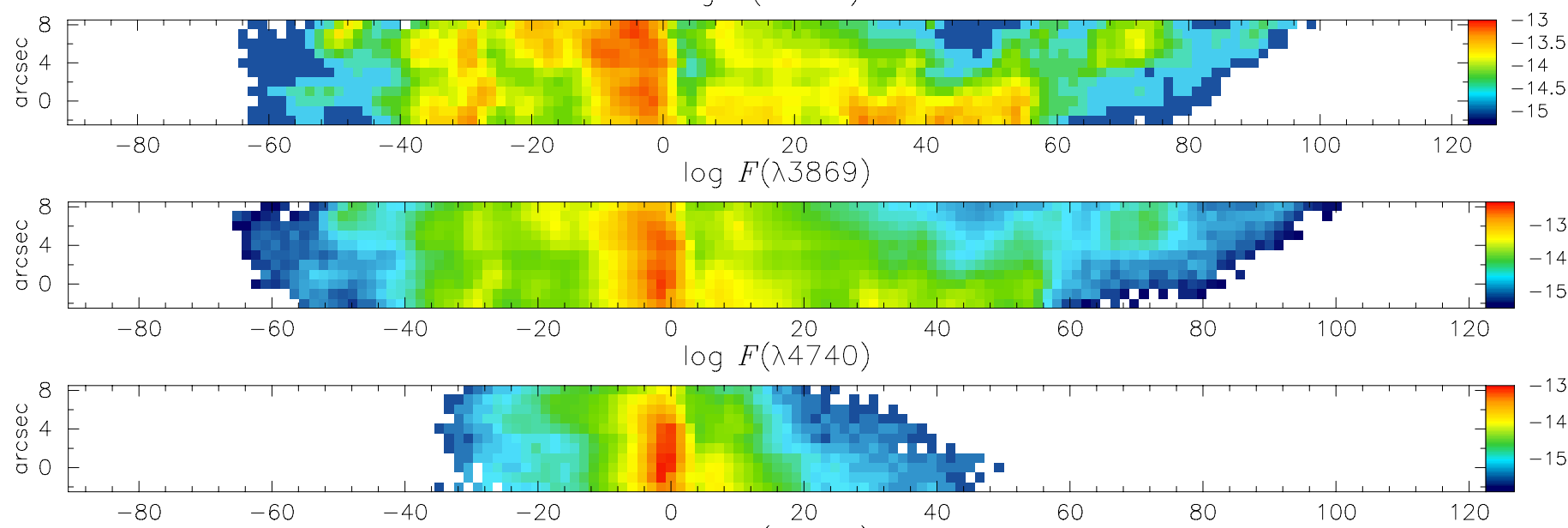

$\log F(\lambda 4686)$
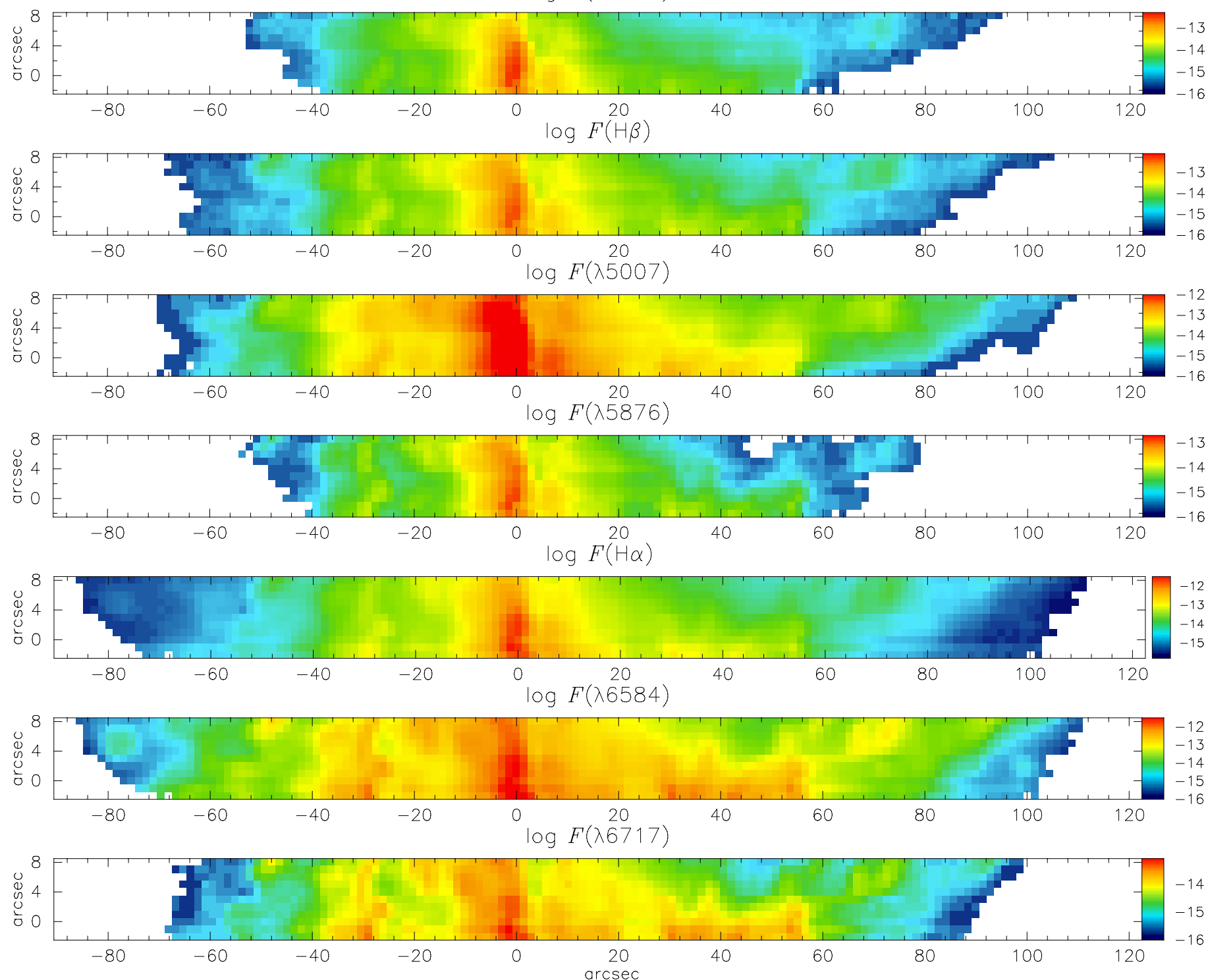

Fig. 2. Logarithmic maps of the observed fluxes (in units of $\mathrm{erg} \mathrm{cm}^{-2} \mathrm{~s}^{-1}$ ) in [O II] $\lambda 3727$, [Ne III] $\lambda 3869, \mathrm{He}$ II $\lambda 4686,[\mathrm{Ar} I V] \lambda 4740, \mathrm{H} \beta$, [O III] $\lambda 5007, \mathrm{He}$ I $\lambda 5876, \mathrm{H} \alpha,[\mathrm{N} \mathrm{II}] \lambda 6584$, and [S II] $\lambda 6717$. In all maps, north is up and east to the left and the coordinates are offset with respect to the position of the central star.

root-mean-square deviation of the continuum. The observed flux $\quad \mathrm{H} \beta$, [O III] $\lambda 5007$, He I $\lambda 5876, \mathrm{H} \alpha$, [N II] $\lambda 6584$, and [S II] $\lambda 6717$

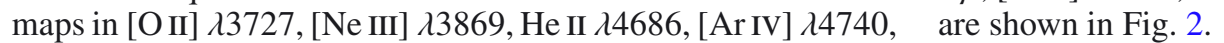




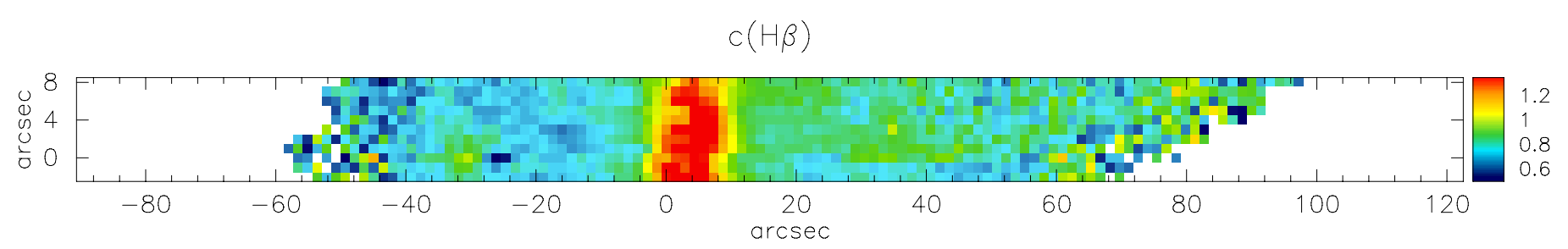

Fig. 3. Map of the logarithmic extinction coefficient $c(\mathrm{H} \beta)$.

\subsection{Extinction}

The observed emission line fluxes with respect to $\mathrm{H} \beta$ were corrected for the effects of the interstellar extinction. The logarithmic extinction coefficient $c(\mathrm{H} \beta)$ was estimated by comparing the $\mathrm{H} \gamma / \mathrm{H} \beta$ and $\mathrm{H} \alpha / \mathrm{H} \beta$ flux ratios measured in each aperture with the case B theoretical ratios computed by Storey \& Hummer (1995) for $T_{\mathrm{e}}=10000 \mathrm{~K}$ and $N_{\mathrm{e}}=1000 \mathrm{~cm}^{-3}$. The Galactic reddening function of Savage \& Mathis (1979) was used.

The $c(\mathrm{H} \beta)$ map presented in Fig. 3 clearly depicts the dust lane crossing the nebula along the north-south direction associated with the molecular disk or torus that gives NGC 6302 its bipolar morphology. The amount of extinction across the nebula is fairly homogeneous in the lobes $(\mathrm{H} \alpha / \mathrm{H} \beta=5.01 \pm 0.34$ and $c(\mathrm{H} \beta)=0.78 \pm 0.10)$, but is much higher in the direction of the circumstellar torus $(\mathrm{H} \alpha / \mathrm{H} \beta=6.94 \pm 0.91$ and $c(\mathrm{H} \beta)=1.23 \pm 0.18)$. We do not confirm previous reports (Rodríguez et al. 1985; Bohigas 1994) that the extinction would be substantially lower in the eastern lobe than in the wertern lobe. We only found a marginal difference in the Balmer ratio with $\mathrm{H} \alpha / \mathrm{H} \beta=4.87 \pm 0.42(c(\mathrm{H} \beta)=0.74 \pm 0.11)$ in the eastern lobe and $\mathrm{H} \alpha / \mathrm{H} \beta=5.11 \pm 0.34(c(\mathrm{H} \beta)=0.81 \pm 0.09)$ in the western lobe.

The area of higher extinction is near the central star position, but displaced to the west. A maximum of $c(\mathrm{H} \beta)=1.69$ $\left(\mathrm{H} \alpha / \mathrm{H} \beta=9.51, A_{\mathrm{H} \alpha}=2.9\right)$ is measured at the position $6^{\prime \prime} \mathrm{W}$ and $5^{\prime \prime} \mathrm{N}$ of the central star. This is consistent with the fact that the torus is not seen exactly edge-on, but with a small inclination of $\approx 15^{\circ}$ (Meaburn et al. 2005; Dinh-V-Trung et al. 2008). Matsuura et al. (2005) mapped the extinction in the central area of NGC 6302 based on the comparison between $\mathrm{H} \alpha$ and $\operatorname{Br} \alpha$ images and a $6 \mathrm{~cm}$ radio continuum map. They found an extinction of $A_{\mathrm{H} \alpha}=3.5-7$, with the highest values towards the disk. These values are higher than those found from the optical Balmer decrement (see, e.g., Tsamis et al. 2003; Groves et al. 2002; Bohigas 1994), indicating that a considerable fraction of the extinction is caused by internal dust.

\subsection{Integrated spectra}

Integrated spectra were obtained by summing the flux contributions in all apertures within the boundary of the $\mathrm{H} \beta$ map. Table A.1 presents the observed and reddening-corrected intensities of the 142 emission lines detected in the integrated spectra. We used a preliminary version of the emission nebula spectral synthesis code X-SSN (Péquignot et al. 2012) to identify faint lines. From the observed Balmer ratios $\mathrm{H} \gamma / \mathrm{H} \beta$ and $\mathrm{H} \alpha / \mathrm{H} \beta$, we obtained a mean extinction coefficient of $c(\mathrm{H} \beta)=1.00 \pm 0.10$. The total observed flux in $\mathrm{H} \beta$ of $2.21 \times 10^{-11} \mathrm{erg} \mathrm{cm}^{-2} \mathrm{~s}^{-1}$ corresponds to $75 \%$ of the integrated flux obtained by Copetti (1990) using narrow-band filter photometry. Thus, although our observations did not cover the whole nebula, a preponderant fraction of $3 / 4$ of the total nebular line emission was included.

\subsection{Excitation properties}

Line ratio maps can provide information about the excitation conditions of the nebula. In Fig. 4, we show the $[\mathrm{N} \mathrm{II}](\lambda 6548+\lambda 6584) / \mathrm{H} \alpha, \quad[\mathrm{S}$ II $](\lambda 6717+\lambda 6731) / \mathrm{H} \alpha$, $[\mathrm{O}$ II $](\lambda 3726+\lambda 3729) / \mathrm{H} \beta$ and $[\mathrm{O}$ III $](\lambda 4959+\lambda 5007) / \mathrm{H} \beta$ maps. To avoid restricting the maps to pixels where $c(\mathrm{H} \beta)$ was measured, the line ratios are not reddening corrected, with the exception of $[\mathrm{O} \mathrm{II}] / \mathrm{H} \beta$. These and other similar maps reflect the stratified structure of photoionized nebulae. Lame \& Ferland (1991) proposed that NGC 6302 might be partially shock-excited by stellar wind. However, Casassus et al. (2000) and Oliva et al. (1996) did not find any indication of shock ionization in this nebula. From the comparison of different line ratios, we tend to conclude that NGC 6302 is essentially a photoionized nebula. To illustrate this, we show in Fig. 5 that the data from NGC 6302 and from supernova remnants occupy separate areas on the plot of $\mathrm{H} \alpha /[\mathrm{S} \mathrm{II}]$ vs. H $\alpha /[\mathrm{N}$ II $]$. However, the effects of possible abundance differences among the objects are not taken into account in this analysis. Furthermore, the presence of very small regions excited by shocks is not discarded. As an example, Phillips et al. (2010) concluded from high spatial resolution data that a small fraction of the emission from some specific regions of NGC 7009 might be due to shock.

\subsection{Electron temperature and density}

Electron temperatures $T_{\mathrm{e}}$ and electron densities $N_{\mathrm{e}}$ were derived from collisional excitation line ratios by numerically solving the equilibrium equations for an $n$-level atom using the IRAF routine temden of the nebular package (Shaw \& Dufour 1995) of STSDAS $^{2}$ with the same atomic parameters used by Krabbe \& Copetti (2005). We determined $T_{\mathrm{e}}$ from [O III] $(\lambda 4959+$ $\lambda 5007) / \lambda 4363$ and $[\mathrm{N}$ II $](\lambda 6548+\lambda 6584) / \lambda 5755$ intensity ratios, and $N_{\mathrm{e}}$ from [S II] $\lambda 6717 / \lambda 6731$ and [Ar IV] $\lambda 4711 / \lambda 4740$. We detected the [Cl III] doublet in some apertures, but we did not use these lines to map the electron density because of large errors associated.

The $[\mathrm{O}$ III] temperature sensor is practically independent of the density assumed. Thus, we derived the electron temperatures $T_{\mathrm{e}}(\mathrm{O}$ III $)$ assuming a constant density of $1000 \mathrm{~cm}^{-3}$. The electron densities $N_{\mathrm{e}}$ (Ar IV) were obtained assuming a constant electron temperature of $15000 \mathrm{~K}$. Errors of less than $5 \%$ are expected if the actual temperature differs by less than $2000 \mathrm{~K}$ of the assumed value. As the lines He I $\lambda 4713$ and [Ar IV] $\lambda 4711$ were not resolved, the contribution of $\mathrm{He}$ I $\lambda 4713$ to the flux measured for [Ar IV] $\lambda 4711$ was estimated as one tenth of the line intensity of He I 14471 (Benjamin et al. 1999).

For the wide range of densities found in NGC 6302, $T_{\mathrm{e}}(\mathrm{N}$ II $)$ shows a significant dependence on the assumed density and

\footnotetext{
2 STSDAS is a product of the Space Telescope Science Institute, which is operated by AURA for NASA.
} 

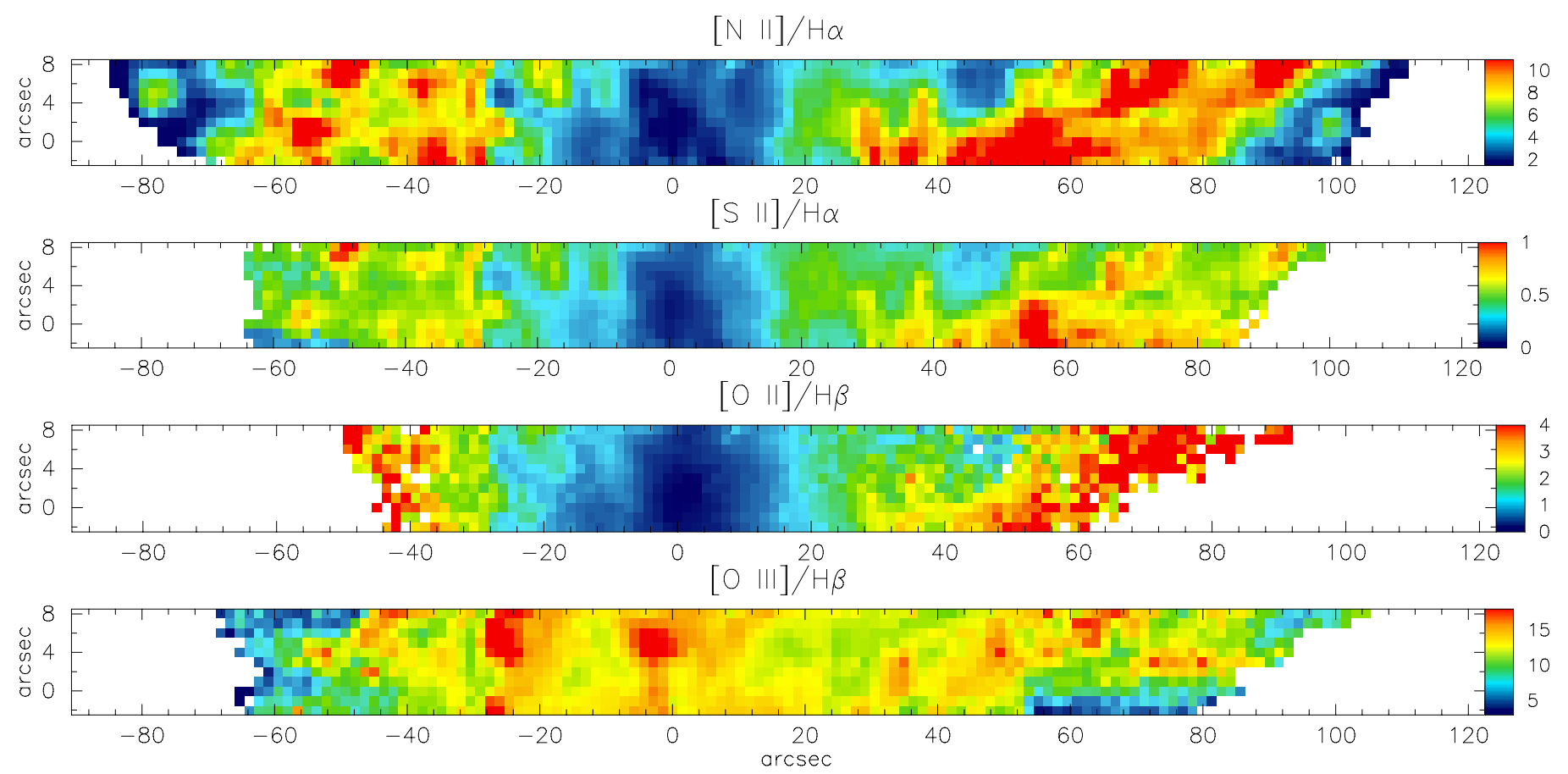

Fig. 4. $[\mathrm{N} I I] / H \alpha$, $[\mathrm{S} \mathrm{II}] / \mathrm{H} \alpha$, $[\mathrm{O} \mathrm{II}] / \mathrm{H} \beta$, and $[\mathrm{O} \mathrm{III}] / \mathrm{H} \beta$ maps.

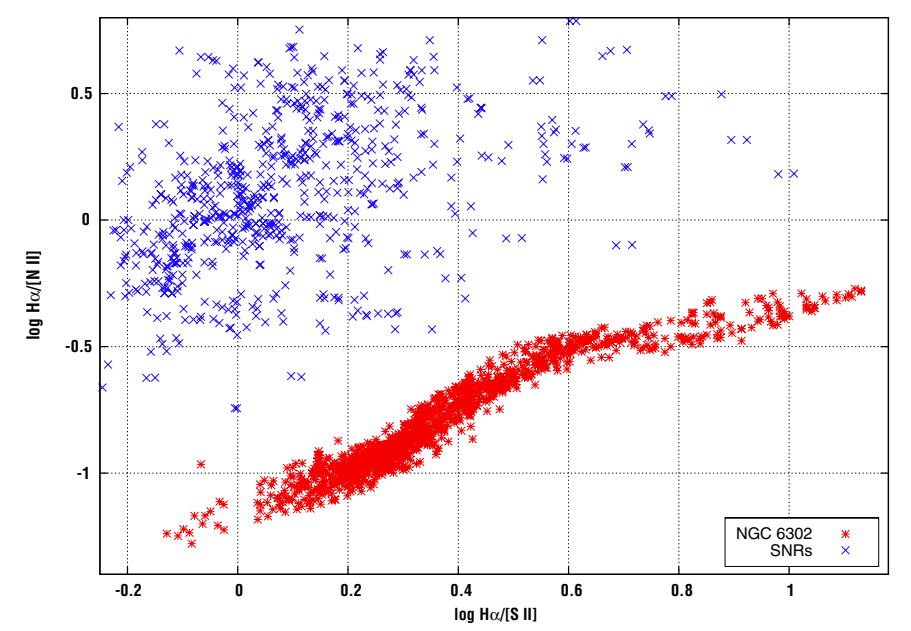

Fig. 5. $\mathrm{H} \alpha /[\mathrm{S} \mathrm{II}]$ vs. $\mathrm{H} \alpha /[\mathrm{N}$ II] for NGC 6302 (this paper) and supernova remnants (data from the literature).

so to derive the $[\mathrm{N}$ II $]$ temperatures and the $[\mathrm{S} \mathrm{II}]$ densities, we followed an interactive procedure. Initially, we calculated the electron densities $N_{\mathrm{e}}(\mathrm{S}$ II $)$ assuming a constant electron temperature of $10000 \mathrm{~K}$. Then, the density values were used to compute $T_{\mathrm{e}}(\mathrm{N}$ II) in each aperture. We iterated until convergence, which was observed after the fifth iteration, to obtain the adopted $N_{\mathrm{e}}\left(\mathrm{S}\right.$ II) and $T_{\mathrm{e}}(\mathrm{N}$ II $)$.

Figure 6 shows the $N_{\mathrm{e}}(\mathrm{S}$ II $), N_{\mathrm{e}}(\mathrm{Ar}$ IV $), T_{\mathrm{e}}(\mathrm{O}$ III $)$, and $T_{\mathrm{e}}(\mathrm{N}$ II $)$ maps. Table 2 presents some non-parametric statistics of the electron density and temperature distributions, including the minimum ( $\min$ ) and maximum ( $\max$ ), the median, the first quartile ( $Q 1$, upper limit of the $25 \%$ lower values), the third quartile ( $Q 3$, lower limit of the $25 \%$ highest values), and the number $N$ of different nebular sections. Table 3 presents the physical conditions determined from the integrated spectra and a comparison with the mean values (weighted by the $\mathrm{H} \beta$ flux) from the temperature maps and with previous values found in the literature.
Table 2. Statistics of the electron density and temperature maps.

\begin{tabular}{llllll}
\hline \hline & \multicolumn{2}{c}{$N_{\mathrm{e}}\left(\mathrm{cm}^{-3}\right)$} & & \multicolumn{2}{c}{$T_{\mathrm{e}}(\mathrm{K})$} \\
\cline { 2 - 3 } \cline { 5 - 6 } & {$[\mathrm{S} \mathrm{II}]$} & {$[$ Ar IV $]$} & & {$[\mathrm{N} \mathrm{II}]$} & {$[\mathrm{O}$ III $]$} \\
\hline $\min$ & 83 & 70 & & 10080 & 13138 \\
$Q 1$ & 393 & 3056 & & 11340 & 16442 \\
median & 963 & 5441 & & 11776 & 17197 \\
$Q 3$ & 1978 & 9750 & & 12499 & 18078 \\
$\max$ & 41634 & 31400 & & 21024 & 26782 \\
$N$ & 1047 & 510 & & 1047 & 813 \\
\hline
\end{tabular}

Table 3. Physical conditions from the integrated spectra, the mean from the temperature maps (weighted by the $\mathrm{H} \beta$ flux) and a comparison with previous works.

\begin{tabular}{lrrrr}
\hline \hline \multirow{2}{*}{ Lines } & \multirow{2}{*}{ Integrated } & \multirow{2}{*}{ Mean } & \multicolumn{2}{c}{ Literature } \\
\cline { 3 - 5 } & & & {$[1]$} & {$[2]$} \\
\hline \multicolumn{5}{c}{$N_{\mathrm{e}}\left(\mathrm{cm}^{-3}\right)$} \\
{$[\mathrm{S} \mathrm{II}]$} & 2900 & 10508 & 12900 & 2183 \\
{$[\mathrm{Cl}$ III $]$} & 4617 & - & 22450 & 13016 \\
{$[\mathrm{Ar}$ IV $]$} & 8690 & 12983 & 14900 & 10630 \\
& & $T_{\mathrm{e}}(\mathrm{K})$ & & \\
{$[\mathrm{O} \mathrm{I}]$} & 6800 & - & - & - \\
{$[\mathrm{S} \mathrm{II}]$} & 13958 & - & 10000 & - \\
{$[\mathrm{O} \mathrm{III}]$} & 17734 & 17390 & 18400 & 17927 \\
{$[\mathrm{Ar}$ V $]$} & 21274 & - & - & - \\
{$[\mathrm{N} \mathrm{II}]$} & 14140 & 12304 & 14225 & 14381 \\
\hline
\end{tabular}

References. [1] Tsamis et al. (2003); [2] Krabbe \& Copetti (2006).

A wide range of values of density is observed with $70 \mathrm{~cm}^{-3} \lesssim$ $N_{\mathrm{e}} \lesssim 41400 \mathrm{~cm}^{-3}$. The density maps of $N_{\mathrm{e}}\left(\mathrm{S}\right.$ II) and $N_{\mathrm{e}}$ (Ar IV) show a similar behavior. The central area of the nebula presents densities above $30000 \mathrm{~cm}^{-3}$. At these high densities, the 

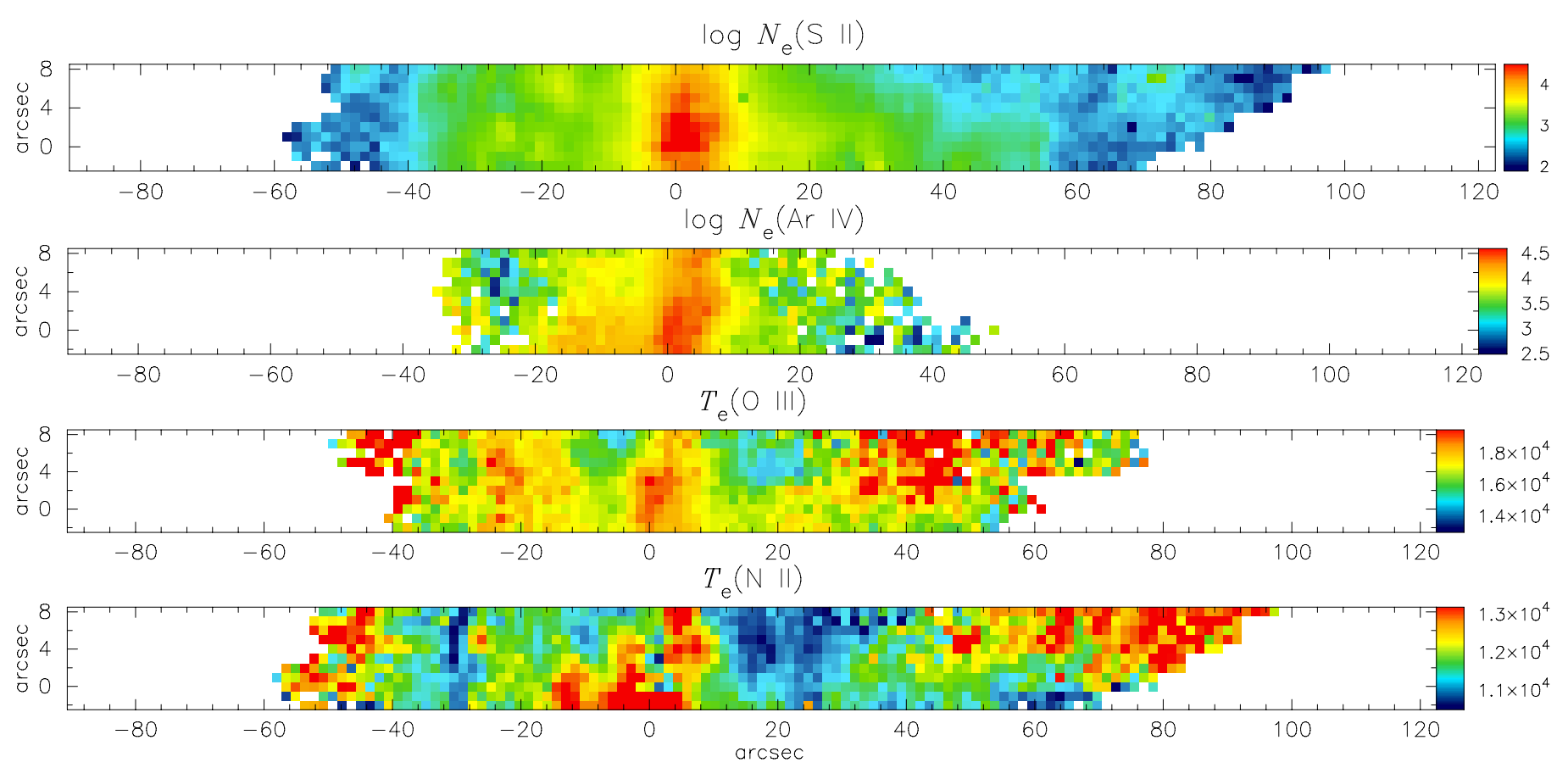

Fig. 6. Maps of the densities $N_{\mathrm{e}}\left(\mathrm{S}\right.$ II) and $N_{\mathrm{e}}(\mathrm{Ar} \mathrm{IV})$ (in units of $\left.\mathrm{cm}^{-3}\right)$ and temperatures $T_{\mathrm{e}}\left(\mathrm{N}\right.$ II) and $T_{\mathrm{e}}(\mathrm{O}$ III) (in units of K).

[S II] line ratio is near saturation, and consequently the derived density values may be uncertain. The maximum values of $N_{\mathrm{e}}(\mathrm{S}$ II $)=41600 \pm 4700 \mathrm{~cm}^{-3}$ and $N_{\mathrm{e}}($ Ar IV $)=31400 \pm$ $420 \mathrm{~cm}^{-3}$ were found at positions less than $2^{\prime \prime}$ from the central star. Both density sensors indicate that the density decreases from the central to the outer regions, but there are some differences that can be seen in the density profiles shown in Fig. 7. The $N_{\mathrm{e}}\left(\mathrm{S}\right.$ II) density falls towards the edges faster than $N_{\mathrm{e}}$ (Ar IV). At the lobes, a uniform low density of less than $1000 \mathrm{~cm}^{-3}$ is observed in the $N_{\mathrm{e}}(\mathrm{S}$ II) map, with the exception of a few knots with 2000-3000 $\mathrm{cm}^{-3}$. Much higher $N_{\mathrm{e}}$ (Ar IV) densities are observed in the lobes. On average, the values of $N_{\mathrm{e}}$ (ArIV) are 2.6 greater than those of $N_{\mathrm{e}}(\mathrm{S}$ II $)$ at the same position. There is an extended area of $20^{\prime \prime}$ to the east of the central star that shows $N_{\mathrm{e}}(\mathrm{Ar}$ IV $) / N_{\mathrm{e}}(\mathrm{S}$ II $) \approx 5-7$. From the integrated spectra, this ratio is about 3 , and $N_{\mathrm{e}}(\mathrm{S}$ II $)<N_{\mathrm{e}}(\mathrm{Cl}$ III $)<N_{\mathrm{e}}(\mathrm{Ar}$ IV $)$, i.e., the density estimated is increasing with the ionization potential of the ion. Thus, all these observations are consistent with the presence of a general internal gradient of the density that decreases towards the outskirts of the nebula. The only area with $N_{\mathrm{e}}(\mathrm{S}$ II $)>N_{\mathrm{e}}(\mathrm{Ar}$ IV $)$ is the densest central region, what may be understood as the consequence of the hampering of the expansion of the nebula towards the molecular torus.

The [N II] and [O III] electron temperature maps (Fig. 6) are almost uniform. Slightly higher $(\approx 10 \%)$ temperatures are found near the central star and at the outskirts of the nebula. The values of the [O III] temperatures are everywhere higher than the [N II] temperatures, with a mean ratio of $T_{\mathrm{e}}(\mathrm{O}$ III $) / T_{\mathrm{e}}(\mathrm{N}$ II $)=$ $1.47 \pm 0.15$. This $50 \%$ difference between the two temperature estimates indicates that there is a significant internal variation of temperature despite the uniformity of the temperature distribution obtained from each sensor. The $\mathrm{N}^{+}$zone is more external than the $\mathrm{O}^{++}$zone, which would indicate a decrease of temperature outwards.

The temperatures derived from the integrated spectra $T_{\mathrm{e}}(\mathrm{N}$ II $)=14140 \mathrm{~K}$ and $T_{\mathrm{e}}(\mathrm{O}$ III $)=17730 \mathrm{~K}$ differ by only $25 \%$ and are in good agreement with the values found in the literature (see Table 3). The weighted mean value of temperature obtained from the maps are $T_{\mathrm{e}}(\mathrm{N}$ II $)=12304 \mathrm{~K}$ (with a dispersion of $1045 \mathrm{~K}$ or $8.5 \%$ ), and $T_{\mathrm{e}}(\mathrm{O} \mathrm{III})=17390 \mathrm{~K}$ (with a dispersion of $1158 \mathrm{~K}$ or $6.7 \%)$. While the mean estimate of $T_{\mathrm{e}}(\mathrm{O}$ III $)$ obtained from the temperature map is similar to that computed from the integrated spectra, a $15 \%$ higher value of $T_{\mathrm{e}}(\mathrm{N}$ II) was obtained from the integrated spectra. This discrepancy may be explained by the dependence of $T_{\mathrm{e}}(\mathrm{N}$ II) on the assumed density.

\subsubsection{Magnitude of the electron temperature fluctuations}

Peimbert (1967) suggested the presence of spatial electron temperature fluctuations to explain the fact that the derived Balmer jump temperatures were significantly lower than the temperatures obtained from forbidden lines. He introduced two parameters to describe the temperature fluctuation over the observed volume of a nebula: the average temperature $T_{0}$ and the mean-square electron temperature fluctuation $t^{2}$ defined as

$t^{2}=\frac{\int\left(T_{\mathrm{e}}-T_{0}\right)^{2} N_{\mathrm{e}} N_{\mathrm{i}} \mathrm{d} V}{T_{0}^{2} \int N_{\mathrm{e}} N_{\mathrm{i}} \mathrm{d} V}$,

with

$T_{0}=\frac{\int T_{\mathrm{e}} N_{\mathrm{e}} N_{\mathrm{i}} \mathrm{d} V}{\int N_{\mathrm{e}} N_{\mathrm{i}} \mathrm{d} V}$,

where $N_{\mathrm{i}}$ is the density of the ion used to measure the temperature. The integrations are computed over the entire volume $V$ of the nebula.

Estimates of $t^{2}$ were obtained assuming that the differences between abundances derived from collisionally excited (CEL) and recombination (RL) lines are due to the presence of these temperature variations. In $\mathrm{PNe}$, these estimatives show high values. As examples, Liu et al. (2006) found $t^{2} \approx 0.14$ for Hf 2-2, and Krabbe \& Copetti (2006) found $t^{2} \approx 0.09$ for NGC 3242 and NGC 7009 to obtain consistent $\mathrm{O}^{++} / \mathrm{H}^{+}$estimates derived from RLs and CELs. 


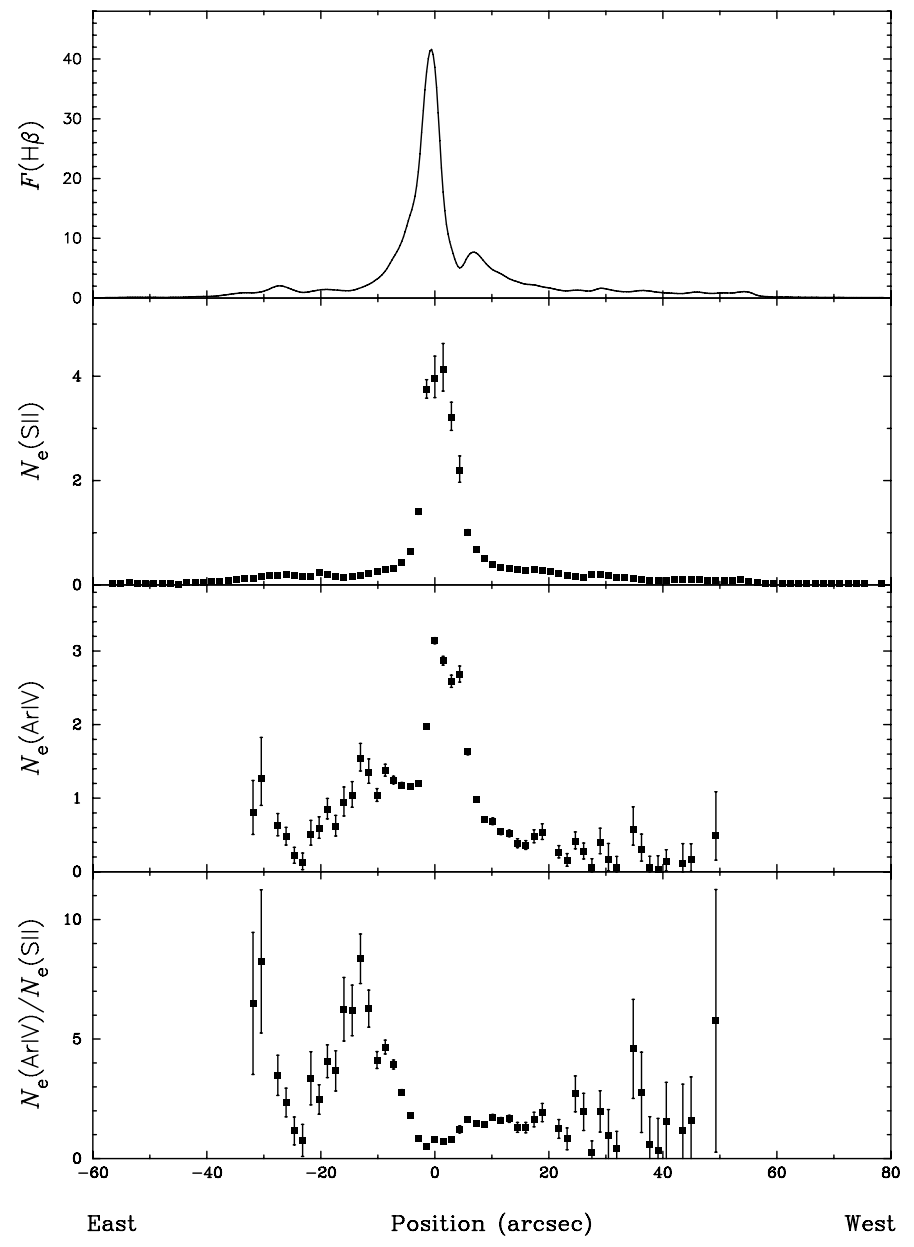

Fig. 7. Spatial profiles of $F(\mathrm{H} \beta)$ (in units of $10^{-14} \mathrm{erg} \mathrm{cm}^{-2} \mathrm{~s}^{-1}$ ), of the densities $N_{\mathrm{e}}\left(\mathrm{S}\right.$ II) and $N_{\mathrm{e}}\left(\mathrm{Ar}\right.$ IV) (in units of $10^{4} \mathrm{~cm}^{-3}$ ), and of the ratio $N_{\mathrm{e}}(\mathrm{Ar}$ IV $) / N_{\mathrm{e}}(\mathrm{S}$ II $)$ along the declination line of the central star.

A discrete estimation of $t^{2}$ on the plane of the sky can be computed from point-to-point measurements of temperature, following the procedure proposed by Liu (1998). A surface temperature fluctuation parameter $t_{\mathrm{s}}^{2}$ is given by

$t_{\mathrm{s}}^{2}(\mathrm{obs})=\frac{\sum_{i}\left(T_{\mathrm{e}}^{i}-T_{0}\right)^{2} F_{i}(\mathrm{H} \beta)}{T_{0}^{2} \sum_{i} F_{i}(\mathrm{H} \beta)}$,

where $T_{\mathrm{e}}^{i}$ and $F_{i}(\mathrm{H} \beta)$ are the temperature and $\mathrm{H} \beta$ flux at the $i$ th aperture, respectively. The summation is over all the observed apertures where the temperature is determined. As part of the temperature variance comes from the measurement errors, a corrected estimate of $t_{\mathrm{s}}^{2}$ can be obtained by $t_{\mathrm{s}}^{2}=t_{\mathrm{s}}^{2}(\mathrm{obs})-t_{\mathrm{errors}}^{2}$, where $t_{\text {errors }}^{2}$ is the quadratic mean of the relative errors of the temperature estimates.

We obtained $t_{\mathrm{S}}^{2}(\mathrm{O}$ III $)=0.0043$ and $t_{\mathrm{S}}^{2}(\mathrm{~N}$ II $)=0.0071$, which are equivalent to the dispersions of $6.6 \%$ and $8.4 \%$, respectively. From long-slit spectra obtained along a single direction, Krabbe \& Copetti (2005) found the similar values of $t_{\mathrm{s}}^{2}(\mathrm{O}$ III $)=0.0050$ and $t_{\mathrm{s}}^{2}(\mathrm{~N}$ II $)=0.0097$ for NGC 6302 and $0.0011 \leq t_{\mathrm{S}}^{2}(\mathrm{O}$ III $) \leq 0.0047$ and $0.0003 \leq t_{\mathrm{s}}^{2}(\mathrm{~N}$ II $) \leq 0.0041$ in nine other PNe. Low values of $t_{\mathrm{s}}^{2}(\mathrm{O}$ III $)=0.0021-0.0025$ were also found in the H II regions NGC 346 and 30 Doradus (Krabbe \& Copetti 2005; Oliveira et al. 2008).

The values of $t_{\mathrm{s}}^{2}$ only provide a lower limit for $t^{2}$, because the temperature calculated for each aperture is an average of the temperatures found along the line of sight. Through numerical simulations, Copetti (2006) found that typically $t_{\mathrm{s}}^{2}(\mathrm{O}$ III $) / t^{2} \approx$ $25 \%$. Thus, we can estimate that $t^{2} \approx 0.017$ in NGC 6302, a value compatible with the predictions of the photoionization models. This level of temperature fluctuation is too low to have any significant impact on the abundance determination from CELs.

\subsection{Nebular abundances}

We constructed maps of the ionic abundances of $\mathrm{N}^{0}, \mathrm{~N}^{+}, \mathrm{O}^{0}$, $\mathrm{O}^{+}, \mathrm{O}^{2+}, \mathrm{S}^{+}, \mathrm{S}^{2+}, \mathrm{Ne}^{2+}, \mathrm{Ar}^{2+}, \mathrm{Ar}^{3+}$, and $\mathrm{Ar}^{4+}$ from CELs using the IRAF task ionic of the package nebular. We adopted a two-zone scheme: $T_{\mathrm{e}}(\mathrm{N}$ II $)$ and $N_{\mathrm{e}}(\mathrm{S}$ II $)$ were used for ions with a low ionization potential $\left(\mathrm{N}^{0}, \mathrm{~N}^{+}, \mathrm{O}^{0}, \mathrm{O}^{+}\right.$, and $\left.\mathrm{S}^{+}\right)$, and $T_{\mathrm{e}}\left(\mathrm{O}\right.$ III) and $N_{\mathrm{e}}(\mathrm{Ar} \mathrm{IV})$ for ions with a high ionization potential $\left(\mathrm{S}^{2+}, \mathrm{O}^{2+}, \mathrm{Ne}^{2+}, \mathrm{Ar}^{2+}, \mathrm{Ar}^{3+}\right.$, and $\left.\mathrm{Ar}^{4+}\right)$. The temperature and density obtained for each aperture were used wherever possible. Otherwise, the mean values for the whole nebula were assumed. We also mapped the $\mathrm{He}^{+}$and $\mathrm{He}^{2+}$ abundances. The $\mathrm{He}^{+}$abundances were determined from the intensities of the He I $\lambda 5876$ line, using the effective recombination coefficients for $\mathrm{H} \beta$ taken from Storey \& Hummer (1995) and for the helium line calculated from the interpolation formula given by Porter et al. (2007). A correction for the collisional contribution to He I $\lambda 5876$ was carried out based on Porter et al. (2007). The $\mathrm{He}^{2+}$ abundances were obtained from the intensities of He II 24686 , using the effective recombination coefficients from Storey \& Hummer (1995). The $T_{\mathrm{e}}(\mathrm{O}$ III) temperatures were adopted in these calculations. The maps of ionic abundances are shown in Figs. 10 and 11. These maps manifest the stratified ionic structure of the nebula. As expected, the abundance maps of ions with similar ionization potential are very similar to each other (e.g., $\mathrm{N}^{+}$and $\mathrm{O}^{0}, \mathrm{He}^{2+}$ and $\mathrm{Ar}^{4+}$ ) and the high ionization potential ions are concentrated around the central star.

We calculated the total abundances using the ionization correction factors ICFs to compensate for the undetected ionic stages for a given element from Kwitter \& Henry (2001) (for Cl) and Kingsburgh \& Barlow (1994) (for the other elements). For $\mathrm{Ne}$ and $\mathrm{K}$, we assume that $\mathrm{Ne} / \mathrm{H}=1.35 \times\left(\mathrm{Ne}^{+} / \mathrm{H}^{+}+\mathrm{Ne}^{2+} / \mathrm{H}^{+}\right)$ (or $\mathrm{Ne} / \mathrm{H}=2.87 \times \mathrm{Ne}^{+} / \mathrm{H}^{+}$when $\mathrm{Ne}^{2+} / \mathrm{H}^{+}$is lacking) and $\mathrm{K} / \mathrm{H}=10.8 \times\left(\mathrm{K}^{3+} / \mathrm{H}^{+}+\mathrm{K}^{4+} / \mathrm{H}^{+}\right)$based on the ionic fractions for $\mathrm{Ne}^{+}, \mathrm{Ne}^{2+}$, and $\mathrm{Ne}^{3+}$ obtained for NGC6302 by Tsamis et al. (2003) and the model for this object by Wright et al. (2011). We also obtained the ionic and the total abundances from the integrated spectra. Table 4 presents some statistics of the ionic abundance maps, including the minimum ("min") and maximum ("max") values, the mean and the standard deviation $(\sigma)$ weighted by the $\mathrm{H} \beta$ flux, the total abundances from the maps, the ionic and total abundances obtained from the integrated spectra, and a comparison with previous abundance determinations found in the literature. The PN NGC 6302 is an extreme case of nitrogen enrichment and the oxygen seems to be depleted by a factor of 2-3 compared to the solar value. The discrepancies between our mean ionic and total abundances and the results reported by other authors can be largely explained by the fact that we considered a much larger portion of the nebula in our analysis.

The total abundances of $\mathrm{He}, \mathrm{N}, \mathrm{O}$, and Ar obtained from the integrated spectra are similar to those derived from the ionic abundance maps within less than $10 \%$ (see the columns labeled "integrated" and "mean" columns in Table 4) despite the different approaches for defining the electron temperature. To calculate the integrated abundances, we adopted a twozone temperature scheme, while the mean ionic abundances are 

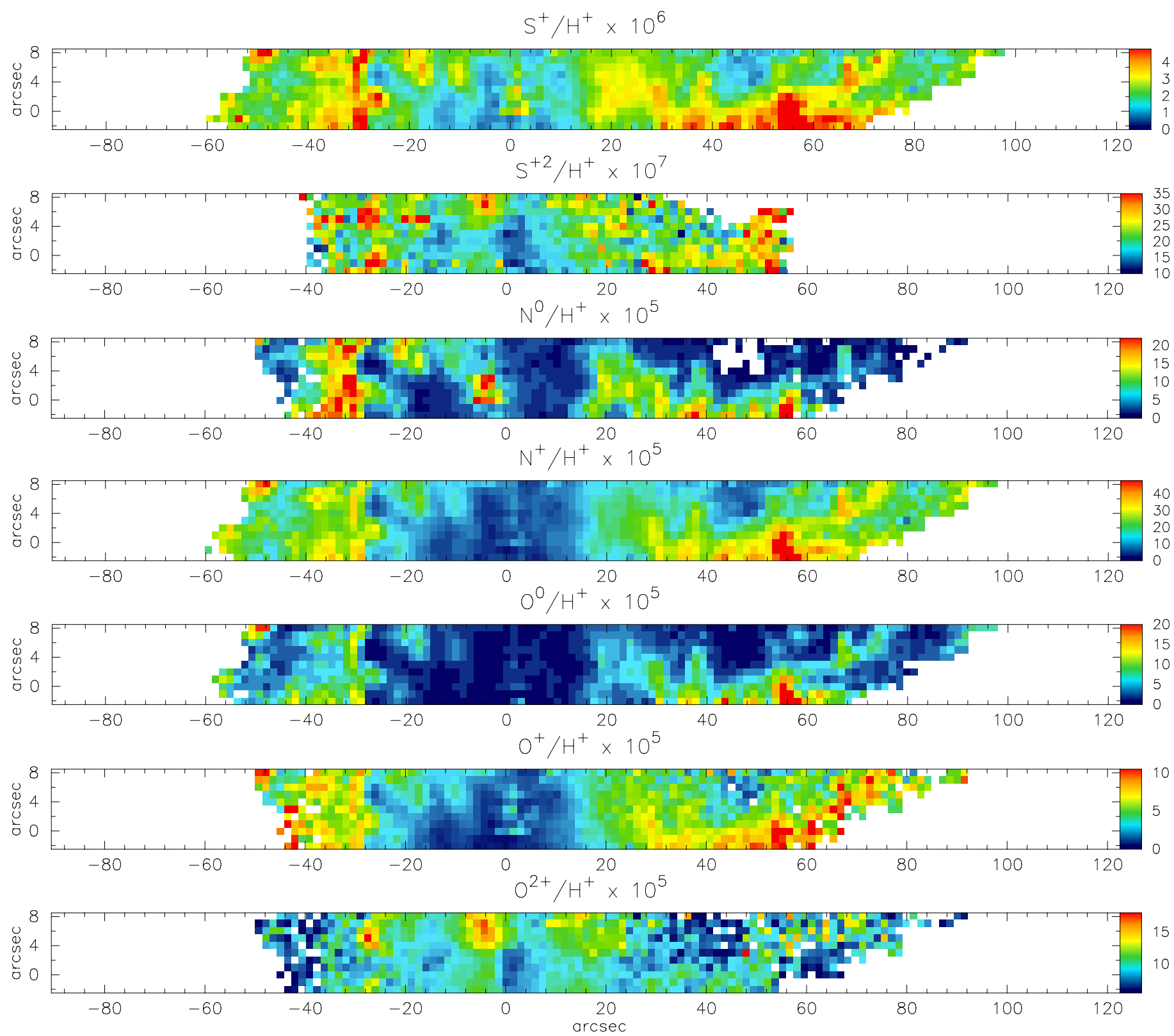

Fig. 8. Maps of ionic abundances.

the average of the values obtained point-to-point. Interestingly, these abundances are also similar to the maximum values of the dominant ionic species of each of these elements. For instance, the maximum ionic abundances $\mathrm{O}^{+} / \mathrm{H}^{+}=1.36 \times 10^{-4}$ and $\mathrm{O}^{2+} / \mathrm{H}^{+}=1.67 \times 10^{-4}$ are similar to the mean and the integrated total oxygen abundances of $\mathrm{O} / \mathrm{H}=1.66 \times 10^{-4}$ and $\mathrm{O} / \mathrm{H}=1.44 \times 10^{-4}$, respectively. This demonstrates the potential of the spatially resolved abundance studies for establishing lower limits of the total abundances based on ionic abundances, for checking the ionization correlation factors, and, in some cases, for determining the total abundances directly from the ionic abundances.

For sulfur, the abundances of the two dominant ions, $\mathrm{S}^{+}$ and $\mathrm{S}^{2+}$, are similar to each other, but are $50 \%$ higher than the total abundance, indicating that the ionization correction factor used,

$\frac{\mathrm{S}}{\mathrm{H}}=\left(1-\left(1-\frac{\mathrm{O}^{+}}{\mathrm{O}}\right)^{3}\right)^{-1 / 3} \frac{\mathrm{S}^{+}+\mathrm{S}^{2+}}{\mathrm{H}^{+}}$, was not entirely appropriate for this nebula. For neon, using the usual ICF (Kingsburgh \& Barlow 1994),

$\frac{\mathrm{Ne}}{\mathrm{H}}=\frac{\mathrm{O}}{\mathrm{O}^{2+}} \frac{\mathrm{Ne}^{2+}}{\mathrm{H}^{+}}$,

the total abundance would be significantly $(\sim 65 \%)$ lower than maximum $\mathrm{Ne}^{2+}$. Therefore, we used instead the fractional ionic abundances of $\mathrm{Ne}^{+}, \mathrm{Ne}^{2+}$, and $\mathrm{Ne}^{3+}$ obtained for NGC 6302 by Tsamis et al. (2003).

\subsection{Apparent overabundances}

We might question whether or not spatial variation of the total abundance of any element can be detected. This is difficult because the observed spectra correspond to the light integrated along the line of sight. For this task, the helium abundance can be considered a good candidate, because the He abundance determination is less sensitive to the electron density and temperature, 
Table 4. Mean ionic and total abundances and comparison with other works.

\begin{tabular}{|c|c|c|c|c|c|c|c|c|c|c|c|c|}
\hline \multirow[b]{2}{*}{ Abundance ratio } & \multicolumn{4}{|c|}{ Map } & \multirow[b]{2}{*}{ Integrated } & \multicolumn{7}{|c|}{ Literature } \\
\hline & $\min$ & $\max$ & mean & $\sigma$ & & {$[1]$} & {$[2]$} & {$[3]$} & [4] & [model] & {$[\mathrm{PN}]$} & [solar] \\
\hline $10^{2} \times \mathrm{He}^{+} / \mathrm{H}^{+}$ & 5.55 & 20.8 & 12.1 & 3.01 & 11.4 & 11.5 & 11.0 & 6.58 & 8.64 & 6.45 & - & - \\
\hline $10^{2} \times \mathrm{He}^{2+} / \mathrm{H}^{+}$ & 1.65 & 8.64 & 5.66 & 1.85 & 5.65 & 6.50 & 6.10 & 6.96 & 6.51 & 4.77 & - & - \\
\hline $10^{2} \times \mathrm{He} / \mathrm{H}$ & - & - & 17.8 & - & 17.0 & 18.0 & 17.1 & 13.5 & 15.2 & 15.3 & 12.9 & 8.60 \\
\hline $10^{5} \times \mathrm{N}^{0} / \mathrm{H}^{+}$ & 0.67 & 27.1 & 7.43 & 5.39 & 3.03 & - & - & - & - & - & - & - \\
\hline $10^{5} \times \mathrm{N}^{+} / \mathrm{H}^{+}$ & 3.90 & 54.0 & 11.2 & 7.93 & 6.86 & 2.60 & 2.90 & 4.48 & 5.86 & 13.4 & - & - \\
\hline $10^{5} \times \mathrm{N} / \mathrm{H}$ & - & - & 71.4 & - & 70.6 & 82.4 & 28.8 & 33.4 & 53.0 & 39.0 & 52.5 & 6.31 \\
\hline $10^{5} \times \mathrm{O}^{0} / \mathrm{H}^{+}$ & 0.51 & 28.0 & 3.00 & 2.90 & 1.64 & - & 1.10 & - & - & - & - & - \\
\hline $10^{5} \times \mathrm{O}^{+} / \mathrm{H}^{+}$ & 0.63 & 13.6 & 2.59 & 1.87 & 1.40 & 0.86 & 1.10 & 0.84 & 1.68 & 30.0 & - & - \\
\hline $10^{5} \times \mathrm{O}^{2+} / \mathrm{H}^{+}$ & 2.81 & 16.7 & 10.3 & 2.20 & 9.60 & 13.0 & 9.00 & 9.18 & 8.80 & 4.17 & - & - \\
\hline $10^{5} \times \mathrm{O} / \mathrm{H}$ & - & - & 16.6 & - & 14.4 & 49.9 & 22.9 & 25.0 & 15.2 & 51.0 & 44.7 & 45.7 \\
\hline $10^{5} \times \mathrm{Ne}^{2+} / \mathrm{H}^{+}$ & 0.75 & 6.84 & 2.56 & 0.74 & 2.44 & - & 7.80 & 2.65 & 2.65 & 1.19 & - & - \\
\hline $10^{5} \times \mathrm{Ne}^{3+} / \mathrm{H}^{+}$ & - & - & - & - & 3.38 & 3.80 & 3.50 & 3.53 & 1.74 & 5.04 & - & - \\
\hline $10^{5} \times \mathrm{Ne} / \mathrm{H}$ & - & - & 7.35 & - & 7.86 & 9.85 & 21.9 & 7.60 & 6.43 & 24.0 & 12.3 & 6.92 \\
\hline $10^{6} \times \mathrm{S}^{+} / \mathrm{H}^{+}$ & 0.86 & 7.59 & 1.99 & 0.93 & 1.03 & 0.20 & 1.30 & 1.04 & 0.51 & 1.28 & - & - \\
\hline $10^{6} \times \mathrm{S}^{2+} / \mathrm{H}^{+}$ & 1.10 & 7.89 & 2.00 & 0.54 & 1.88 & 1.96 & 2.20 & 1.57 & 1.60 & 1.98 & - & - \\
\hline $10^{6} \times \mathrm{S} / \mathrm{H}$ & - & - & 5.42 & - & 4.54 & 6.21 & 7.94 & 5.59 & 3.17 & 25.0 & 8.13 & 21.4 \\
\hline $10^{7} \times \mathrm{Ar}^{2+} / \mathrm{H}^{+}$ & 4.42 & 17.8 & 10.1 & 1.89 & 9.24 & 8.00 & 20.0 & 6.51 & - & 7.10 & - & - \\
\hline $10^{7} \times \mathrm{Ar}^{3+} / \mathrm{H}^{+}$ & 1.30 & 7.94 & 5.71 & 1.40 & 7.63 & 28.3 & 8.60 & 7.75 & - & 3.39 & - & - \\
\hline $10^{7} \times \mathrm{Ar}^{4+} / \mathrm{H}^{+}$ & 0.48 & 7.84 & 5.09 & 1.91 & 3.40 & 15.9 & 3.60 & 4.67 & - & - & - & - \\
\hline $10^{7} \times \mathrm{Ar} / \mathrm{H}$ & - & - & 24.8 & - & 22.5 & 85.0 & 60.2 & 21.9 & - & 120 & 26.3 & 15.1 \\
\hline $10^{5} \times \mathrm{C}^{0} / \mathrm{H}^{+}$ & - & - & - & - & 0.24 & - & - & - & - & - & - & - \\
\hline $10^{5} \times \mathrm{C} / \mathrm{H}$ & - & - & - & - & - & 10.1 & 6.00 & 7.70 & - & 22.0 & 30.2 & 25.7 \\
\hline $10^{8} \times \mathrm{K}^{3+} / \mathrm{H}^{+}$ & - & - & - & - & 4.02 & 5.51 & 1.90 & - & - & - & - & - \\
\hline $10^{8} \times \mathrm{K}^{4+} / \mathrm{H}^{+}$ & - & - & - & - & 4.76 & - & - & - & - & - & - & - \\
\hline $10^{8} \times \mathrm{K} / \mathrm{H}$ & - & - & - & - & $85:$ & 21.0 & 85.0 & - & - & 50.0 & - & 13.5 \\
\hline $10^{8} \times \mathrm{Cl}^{2+} / \mathrm{H}^{+}$ & - & - & - & - & 3.91 & 3.40 & 3.70 & 2.76 & 2.84 & 11.3 & - & - \\
\hline $10^{8} \times \mathrm{Cl}^{3+} / \mathrm{H}^{+}$ & - & - & - & - & 3.59 & 13.5 & 2.10 & - & - & - & - & - \\
\hline $10^{8} \times \mathrm{Cl} / \mathrm{H}$ & - & - & - & - & 11.2 & 39.1 & 34.0 & 9.80 & 5.60 & 25.0 & - & 31.6 \\
\hline $10^{8} \times \mathrm{Ca}^{4+} / \mathrm{H}^{+}$ & - & - & - & - & 1.68 & 3.83 & 2.50 & - & - & - & - & - \\
\hline $10^{8} \times \mathrm{Ca} / \mathrm{H}$ & - & - & - & - & 7.40 & 22.8 & - & - & - & - & - & 229 \\
\hline
\end{tabular}

References. [1] Aller et al. (1981); [2] Pottasch \& Beintema (1999); [3] Tsamis et al. (2003); [4] Krabbe \& Copetti (2006); [model] by Wright et al. (2011); [PN] mean of Type I PN by Kingsburgh \& Barlow (1994); [solar] by Grevesse \& Sauval (1998).

and the emission lines are strong. The total helium abundance map was obtained by summing the $\mathrm{He}^{+} / \mathrm{H}^{+}$and $\mathrm{He}^{2+} / \mathrm{H}^{+}$abundance maps and is shown in Fig. 12. As expected, areas near the edges of the nebula tend to show lower values of this abundance ratio, because these are the regions where the contribution of neutral He may be significant. Excluding these areas, the dispersion of $\left(\mathrm{He}^{+}+\mathrm{He}^{2+}\right) / \mathrm{H}^{+}$is only $8 \%$. The individual maps of $\mathrm{He}^{+} / \mathrm{H}^{+}$and $\mathrm{He}^{2+} / \mathrm{H}^{+}$show much higher variations, which were mostly canceled out by summing the two maps. The remaining systematic variation of $\left(\mathrm{He}^{+}+\mathrm{He}^{2+}\right) / \mathrm{H}^{+}$is probably a meaningless residual of this sum.

The ratio of ionic abundances $\mathrm{N}^{+} / \mathrm{O}^{+}$, also shown in Fig. 12, is often taken as an estimate of the total abundance N/O. In the south of the nebula, there is an extended area where values $30 \%$ to $60 \%$ higher than the mean are observed. Gonçalves et al. (2003) obtained homogeneous elemental abundances along NGC 7009. However, they found some outer structures with an apparent overabundance of $\mathrm{N}$ (by a factor of \$2).
They attributed this difference to possible errors in the ICFs. Alexander \& Balick (1997) showed that, in spatially resolved analysis, the errors due to the ICFs can be high, mainly for $\mathrm{N}, \mathrm{Ne}$, and Ar, and can lead to overestimated $\mathrm{N} / \mathrm{O}$ variations in bipolar nebulae. To investigate this, Gonçalves et al. (2006) used three-dimensional photoionization models, whose constraints were the observational data of Gonçalves et al. (2003). They predicted ratios of $\left(\mathrm{N}^{+} / \mathrm{N}\right) /\left(\mathrm{O}^{+} / \mathrm{O}\right)$ significantly lower than unity (up to $40 \%$ ). Thus, the usual assumption that $\mathrm{N}^{+} / \mathrm{N}=\mathrm{O}^{+} / \mathrm{O}$ may not be appropriate for all parts of the nebula. A similar problem with the ICFs could be the cause of the apparent overabundances found in some regions of NGC 6302.

\subsection{Comparison with the 3D model of Wright et al. (2011)}

Wright et al. (2011) presented a 3D photoionization model of NGC 6302 based on data compiled from the literature. The final best-fitting model was achieved with a characteristic density for 

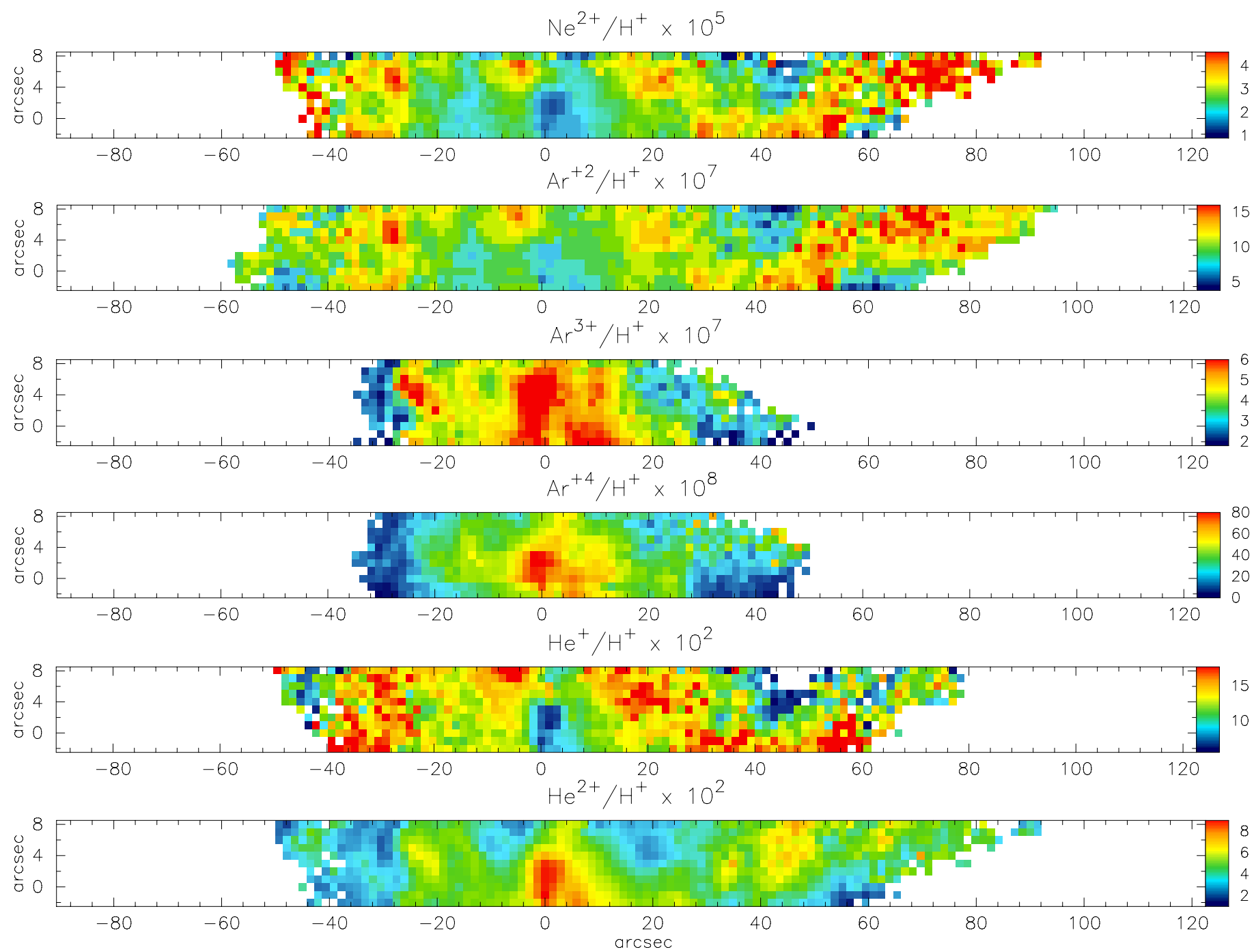

Fig. 9. Maps of ionic abundances.

the circumstellar disk of $80000 \mathrm{~cm}^{-3}$. This was the most influential parameter of the model. The lobes were modeled with a constant density of $2000 \mathrm{~cm}^{-3}$, and an uncertainty of $\pm 50 \%$ was estimated for this value. To improve the fitting of some line ratios sensitive to the electron density and temperature, they assumed the presence in the inner parts of the lobes of a 12" area with a high density of $20000 \mathrm{~cm}^{-3}$ and other inhomogeneous densities, which were simulated using 5-50 knots distributed randomly across the nebula with overdensities up to a factor of 3. Although based on limited spatially resolved information, the model density structure reproduces remarkably well the main characteristics of the electron density maps of $N_{\mathrm{e}}(\mathrm{S}$ II $)$ and $N_{\mathrm{e}}$ (Ar IV) (Fig. 2).

With regard to the thermal structure of the nebula, the model predicts a monotonic radial decrease in electron temperature away from the center. The temperature estimates from integrated line ratios of different ions shown in Table 3 indicate that the temperature tends to increase with ionization potential of the ions, in agreement with the temperature gradient predicted by the model. The maps of $T_{\mathrm{e}}\left(\mathrm{O}\right.$ III) and $T_{\mathrm{e}}(\mathrm{N}$ II) (Fig. 6) show higher temperatures near the central star, as predicted by the model, but also at the outskirts of the nebula. Moreover, Wright et al. (2011) found average temperatures of $12000 \mathrm{~K}$ over the entire nebula, $19400 \mathrm{~K}$ in the lobes, and $3800 \mathrm{~K}$ in the circumstellar disk. The mean electron temperatures weighted by the $\mathrm{N}^{+}$and $\mathrm{O}^{2+}$ ions were, respectively, $4800 \mathrm{~K}$ and $10900 \mathrm{~K}$ for the circumstellar disk, and $8480 \mathrm{~K}$ and $10360 \mathrm{~K}$ for the bipolar lobes. These values are much lower than those found in our temperature maps and also lower than the temperatures estimated via the integrated spectra. Consequently, some ionic abundance ratios derived from the model differ greatly from our results (Table 4), especially those of low ionization ions. For instance, Wright et al. (2011) obtained a ratio $\mathrm{O}^{+} / \mathrm{H}^{+}$about 8 times higher than our mean value, while the estimates for $\mathrm{O}^{2+} / \mathrm{H}^{+}$differ by less than $10 \%$. For the total abundances used by Wright et al. (2011) as model parameters, the highest discrepancies are verified for $\mathrm{Ar} / \mathrm{H}, \mathrm{Ne} / \mathrm{H}$, and $\mathrm{O} / \mathrm{H}$, which are higher than our values by factors of about 10,6 , and 3 , respectively.

\section{Conclusions}

Through spatially resolved long-slit spectroscopy, we studied the physical and chemical conditions of the PN NGC 6302. Data from 11 parallel and equally spaced directions were obtained, allowing the construction of maps of line fluxes, line ratios, electron density and temperature, and ionic abundances with a spatial resolution of $1.45^{\prime \prime} \times 1^{\prime \prime}$ over an area of $11^{\prime \prime} \times 250^{\prime \prime}$. 

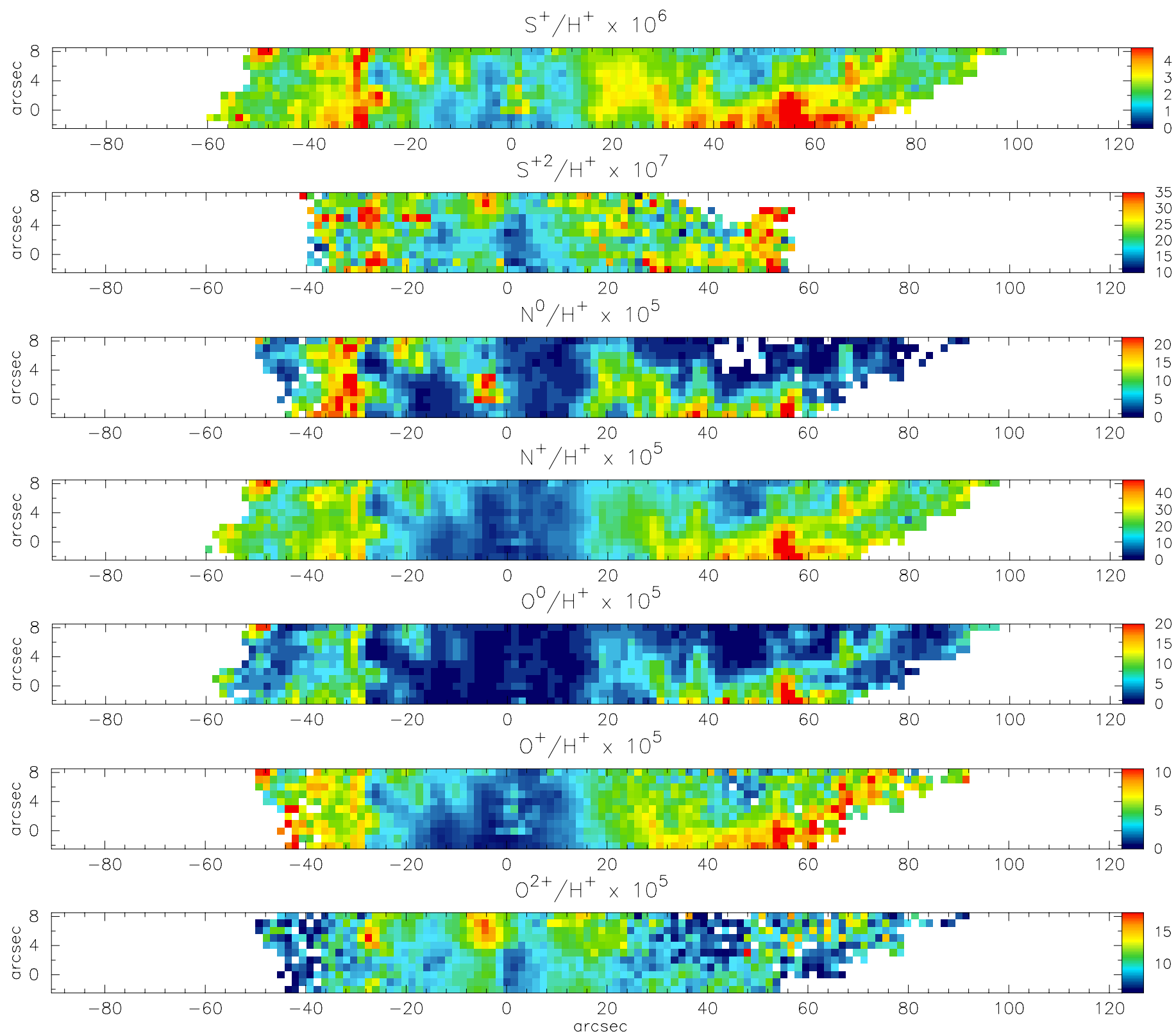

Fig. 10. Maps of ionic abundances.

Integrated spectra were also obtained and 142 lines were identified. The main results are the following.

1. The $c(\mathrm{H} \beta)$ extinction map shows a dust lane crossing the nebula along the north-south direction associated with the molecular disk or torus that gives NGC 6302 its bipolar morphology. The extinction is fairly homogeneous in the lobes $(c(\mathrm{H} \beta)=0.78 \pm 0.10)$, but it is much higher in the direction of the circumstellar torus $(c(\mathrm{H} \beta)=1.23 \pm 0.18)$. The area of higher extinction is near, but displaced to the west, of the central star position. This is consistent with the fact that the torus is not seen exactly edge-on, but with a small inclination angle.

2. The [S II] and [Ar IV] electron density maps indicate the presence of a steep internal gradient with the density decreasing towards the outskirts of the nebula. The density peaks above $40000 \mathrm{~cm}^{-3}$ at the central area and drops below $100 \mathrm{~cm}^{-3}$ at the edges of the nebula.
3. The $[\mathrm{N} \mathrm{II}]$ and $[\mathrm{O}$ III $]$ electron temperature maps are almost uniform. Slightly higher $(\approx 10 \%)$ temperatures are found near the central star and at the outskirts of the nebula. The [O III] temperature is everywhere higher than the [N II] temperature, with a mean ratio of $T_{\mathrm{e}}(\mathrm{O}$ III $) / T_{\mathrm{e}}(\mathrm{N}$ II $)=1.47 \pm$ 0.15 , indicating that there is a significant internal variation of temperature despite the uniformity of the temperature distribution obtained from each sensor. Among the five different temperature estimates obtained from the integrated spectra, we observed a correlation between the temperature estimate and the ionization potential of the ion, which indicates the presence of an internal radial decrease in electron temperature.

4. From the distributions of the $[\mathrm{N} \mathrm{II}]$ and $[\mathrm{O}$ III $]$ electron temperatures on the plane of the sky, we obtained the surface temperature fluctuation parameters $t_{\mathrm{s}}^{2}(\mathrm{O}$ III $)=0.0043$ and $t_{\mathrm{s}}^{2}(\mathrm{~N}$ II $)=0.0071$, which are equivalent to dispersions of $6.6 \%$ and $8.4 \%$, respectively. Based on numerical 

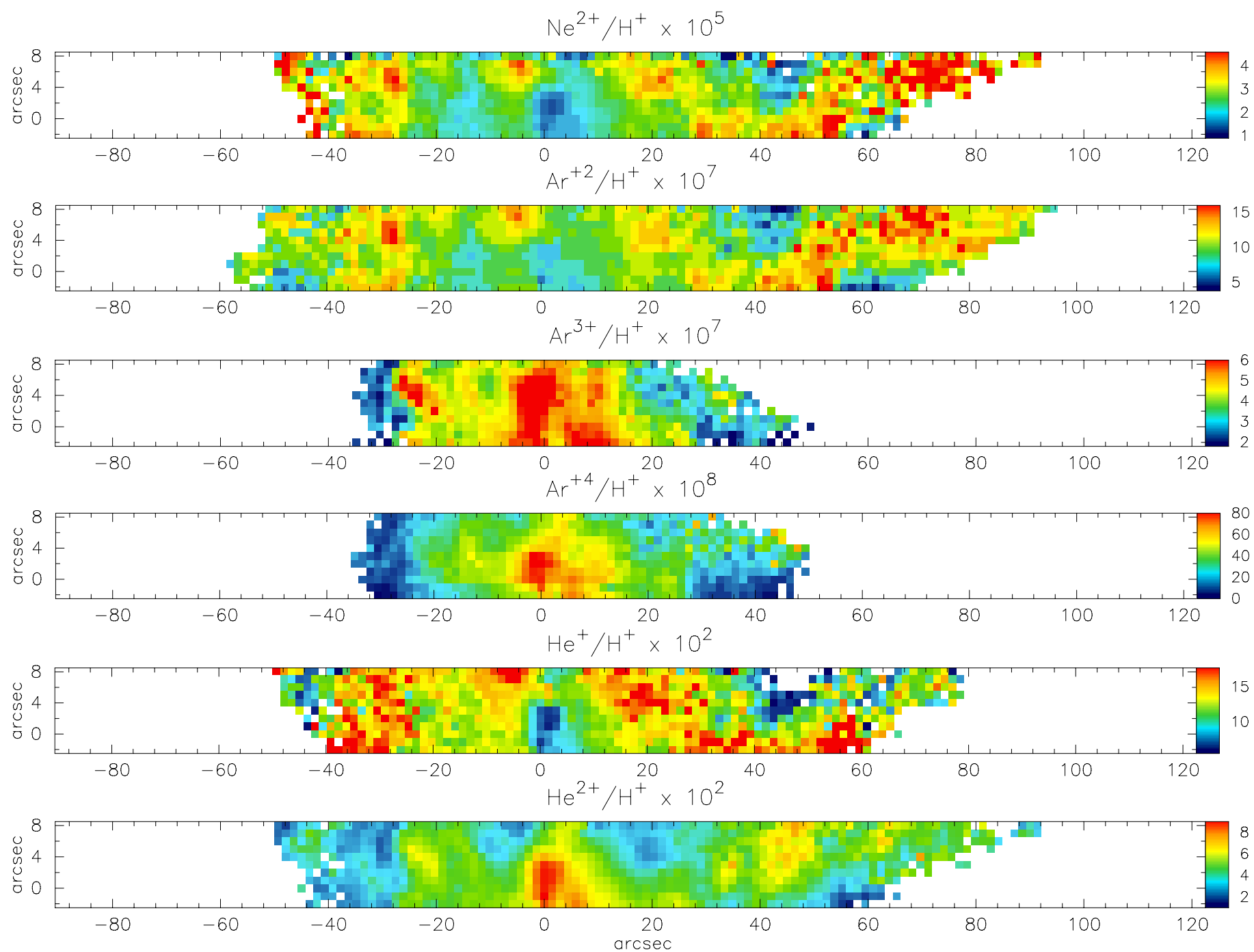

Fig. 11. Maps of ionic abundances.
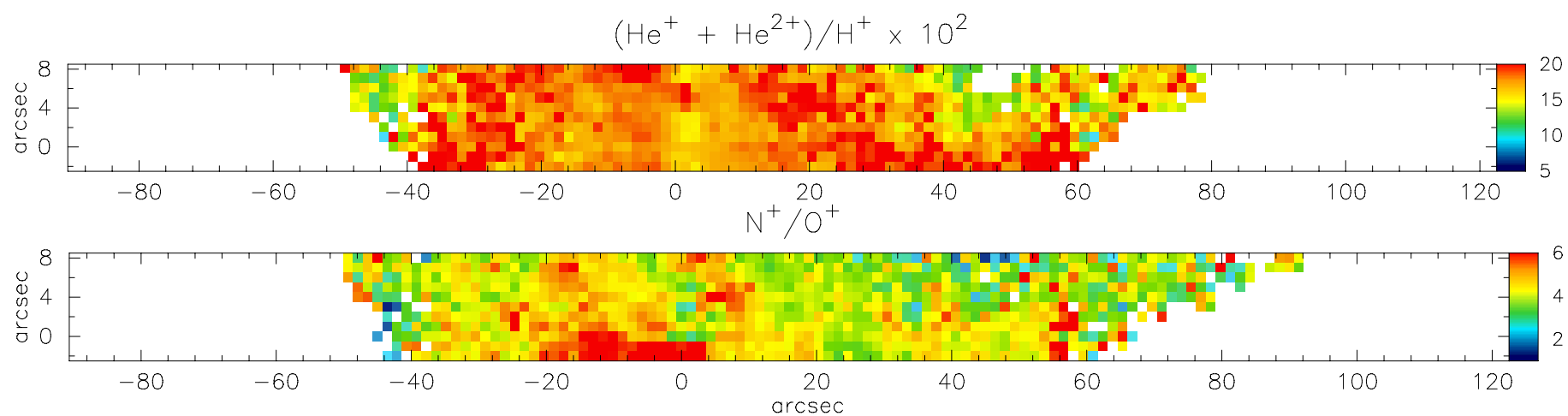

Fig. 12. Maps of the ionic abundances $\left(\mathrm{He}^{+}+\mathrm{He}^{2+}\right) / \mathrm{H}^{+}$and $\mathrm{N}^{+} / \mathrm{O}^{+}$.

simulations, we estimate a 3D temperature fluctuation parameter of $t^{2} \approx 0.017$ in NGC 6302, a value compatible with the predictions of the photoionization models and too small to have any significant impact on the abundance determination from collisionally excited lines.

5. We constructed maps of the ionic abundances of $\mathrm{He}^{+}, \mathrm{He}^{2+}$, $\mathrm{N}^{0}, \mathrm{~N}^{+}, \mathrm{O}^{0}, \mathrm{O}^{+}, \mathrm{O}^{2+}, \mathrm{S}^{+}, \mathrm{S}^{2+}, \mathrm{Ne}^{2+}, \mathrm{Ar}^{2+}, \mathrm{Ar}^{3+}$, and $\mathrm{Ar}^{4+}$ and derived the total abundances. We also obtained the ionic and the total abundances from the integrated spectra. For many elements, the total abundances obtained from the integrated spectra are similar to those derived from the ionic abundance maps and are also similar to the maximum abundances of the dominant ionic species of each of these elements. This demonstrates the potential of the spatially resolved abundance studies for establishing lower limits of the total abundances based on ionic abundances, for checking the ionization correlation factors, and, in some cases, for determining the total abundances directly from the ionic abundances.

Acknowledgements. This work was supported by the Brazilian agencies CAPES and CNPq. A. C. Krabbe thanks for the support of FAPESP, process 2010/01490-3. 
A. B. Rauber et al.: Spectroscopic mapping of the planetary nebula NGC 6302

\section{References}

Alexander, J., \& Balick, B. 1997, ApJ, 114, 713

Aller, L. H., Ross, J. E., Omara, B. J., \& Keyes, C. D. 1981, MNRAS, 197, 95

Ashley, M. C. B., \& Hyland, A. R. 1988, ApJ, 331, 532

Benjamin, R. A., Skillman, E. D., \& Smits, D. P. 1999, ApJ, 514, 307

Bohigas, J. 1994, A\&A, 288, 617

Casassus, S., Roche, P. F., \& Barlow, M. J. 2000, MNRAS, 314, 657

Copetti, M. V. F. 1990, PASP, 102, 77

Copetti, M. V. F. 2006, A\&A, 453, 943

Dinh-V-Trung, Bujarrabal, V., Castro-Carrizo, A., Lim, J., \& Kwok, S. 2008, ApJ, 673, 934

Feibelman, W. A. 2001, ApJ, 550, 785

Gonçalves, D. R., Corradi, R. L. M., Mampaso, A., \& Perinotto, M. 2003, ApJ, 597,975

Gonçalves, D. R., Ercolano, B., Carnero, A., Mampaso, A., \& Corradi, R. L. M. 2006, MNRAS, 365, 1039

Grevesse, N., \& Sauval, A. J. 1998, Space Sci. Rev., 85, 161

Groves, B., Dopita, M. A., Williams, R. E., \& Hua, C. 2002, PASA, 19, 425

Kingdon, J., \& Ferland, G. J. 1995, AJ, 442, 714

Kingsburgh, R. L., \& Barlow, M. J. 1994, MNRAS, 271, 257

Krabbe, A. C., \& Copetti, M. V. F. 2005, A\&A, 443, 981

Krabbe, A. C., \& Copetti, M. V. F. 2006, A\&A, 450, 159

Kwitter, K. B., \& Henry, R. B. C. 2001, ApJ, 562, 804

Liu, X.-W. 1998, MNRAS, 295, 699

Liu, X.-W., Storey, P. J., Barlow, M. J., et al. 2000, MNRAS, 312, 585

Liu, X.-W., Barlow, M. J., Zhang, Y., Bastin, R. J., \& Storey, P. J. 2006, MNRAS, 368,1959

Leal-Ferreira, M. L., Gonçalves, D. R., Monteiro, H., \& Richards, J. W. 2011, MNRAS, 411, 1395
Lame, N. J., \& Ferland, G. J. 1991, ApJ, 367, 208

Matsuura, M., Zijlstra, A. A., Molster, F. J., et al. 2005, MNRAS, 359, 383

Meaburn, J., López, J. A., Steffen, W., Graham, M. F., \& Holloway, A. J. 2005, AJ, 130, 2303

Monteiro, H., Schwarz, H. E., Gruenwald, R., \& Heathcote, S. 2004, AJ, 609, 194

Oliveira, V. A., Copetti, M. V. F., \& Krabbe, A. C. 2008, A\&A, 492, 463

Peimbert, M. 1967, ApJ, 150, 825

Peimbert, \& M., Torres-Peimbert, S. 1983, in Type I Planetary Nebulae, ed., D. R. Flower, IAU Symp., 103, 233

Péquignot, D., Morisset, C., \& Casassus, S. 2012, IAU Symp., 283, 470

Peretto, N., Fuller, G., Zijlstra, A., Patel, N. 2007, A\&A, 473, 207

Pottasch, S. R., \& Beintema, D. A. 1999, A\&A, 347, 975

Rodriguez, L. F., Garcia-Barreto, J. A., Canto, J., et al. 1985, MNRAS, 215, 353

Sabbadin, F., Minello, S., \& Bianchini, A. 1977, A\&A, 60, 147

Savage, B. D., \& Mathis J. S. 1979, ARA\&A, 17, 73

Shaw, R. A., \& Dufour, R. J. 1995, PASP, 107, 896

Storey, P. J., \& Hummer, D. G. 1995, MNRAS, 272, 41

Szyszka, C., Zijlstra, A. A., \& Walsh, J. R. 2011, MNRAS, 416, 715

Szyszka, C., Walsh, J. R., Zijlstra, A. A., \& Tsamis, Y. G. 2009, AJ, 707, 32

Oliva, E., Pasquali, A., Reconditi, M. 1996, A\&A, 305, 21

Phillips, J. P., Cuesta, L. C., \& Ramos-Larios, G. 2010, MNRAS, 409, 881

Porter, R. L., Ferland, G. J., MacAdam, K. B. 2007, AJ, 657, 327

Tsamis, Y. G., Barlow, M. J., Liu,X.-W, Danziger, I. J., \& Storey, P. J. 2003, MNRAS, 345, 186

Tsamis, Y. G., Walsh, J. R., Péquignot, D., Barlow, M. J., \& Danziger, I. J. 2008, MNRAS, 386, 22

Wright, N. J., Barlow, M. J., Ercolano, B., \& Rauch, T. 2011, MNRAS, 418, 370 


\section{Appendix A}

Table A.1. Observed $F(\lambda)$ and dereddened $I(\lambda)$ line intensities (on a scale $F(\mathrm{H} \beta)=100)$ and the reddening function $f(\lambda)$.

\begin{tabular}{|c|c|c|c|}
\hline Line & $f(\lambda)$ & $F(\lambda)$ & $I(\lambda)$ \\
\hline He I $\lambda 3554$ & 0.318 & $0.38 \pm 0.01$ & $0.69 \pm 0.02$ \\
\hline $\mathrm{He} \mathrm{I}+[\mathrm{Fe}$ VII $] \lambda 3587$ & 0.310 & $0.62 \pm 0.03$ & $1.09 \pm 0.04$ \\
\hline He I $\lambda 3614$ & 0.303 & $0.40 \pm 0.01$ & $0.71 \pm 0.02$ \\
\hline Не I $\lambda 3634$ & 0.298 & $0.66 \pm 0.03$ & $1.14 \pm 0.04$ \\
\hline H17 $\lambda 3698$ & 0.282 & $0.41 \pm 0.01$ & $0.69 \pm 0.02$ \\
\hline $\mathrm{H} 16$ + He I $\lambda 3704$ & 0.280 & $0.72 \pm 0.03$ & $1.20 \pm 0.05$ \\
\hline H15 $\lambda 3712$ & 0.278 & $0.76 \pm 0.02$ & $1.27 \pm 0.04$ \\
\hline [O II] $\lambda 3727$ & 0.275 & $55.5 \pm 0.1$ & $92.0 \pm 0.3$ \\
\hline $\mathrm{H} 12 \lambda 3750$ & 0.269 & $1.50 \pm 0.03$ & $2.47 \pm 0.05$ \\
\hline$[\mathrm{Fe}$ VII $]+$ O III $\lambda 3759$ & 0.267 & $2.18 \pm 0.04$ & $3.56 \pm 0.06$ \\
\hline H11 $\lambda 3770$ & 0.264 & $1.95 \pm 0.02$ & $3.17 \pm 0.03$ \\
\hline He I $\lambda 3782$ & 0.262 & $0.64 \pm 0.02$ & $1.04 \pm 0.04$ \\
\hline H10 $\lambda 3798$ & 0.258 & $2.60 \pm 0.01$ & $4.17 \pm 0.02$ \\
\hline He II $\lambda 3813$ & 0.255 & $0.26 \pm 0.01$ & $0.41 \pm 0.02$ \\
\hline He I $\lambda 3820$ & 0.253 & $1.02 \pm 0.01$ & $1.62 \pm 0.02$ \\
\hline H9 $\lambda 3835$ & 0.249 & $4.02 \pm 0.01$ & $6.36 \pm 0.02$ \\
\hline [Ne III] $\lambda 3869$ & 0.242 & $98.28 \pm 0.05$ & $153.4 \pm 0.2$ \\
\hline $\mathrm{He}$ I $+\mathrm{H} 8 \lambda 3889$ & 0.237 & $14.71 \pm 0.01$ & $22.77 \pm 0.03$ \\
\hline He II $\lambda 3923$ & 0.229 & $0.77 \pm 0.03$ & $1.17 \pm 0.04$ \\
\hline$[\mathrm{Ne}$ III] + H7 $\lambda 3968$ & 0.220 & $45.78 \pm 0.02$ & $68.66 \pm 0.07$ \\
\hline Не г $\lambda 4009$ & 0.211 & $0.347 \pm 0.009$ & $0.51 \pm 0.01$ \\
\hline Не I $\lambda 4026$ & 0.207 & $2.92 \pm 0.01$ & $4.28 \pm 0.02$ \\
\hline [S II] $\lambda 4069$ & 0.198 & $9.06 \pm 0.02$ & $13.05 \pm 0.03$ \\
\hline [S II] $\lambda 4076$ & 0.196 & $3.04 \pm 0.01$ & $4.37 \pm 0.02$ \\
\hline $\mathrm{H} \delta \lambda 4101$ & 0.191 & $19.85 \pm 0.02$ & $28.21 \pm 0.04$ \\
\hline $\mathrm{He} \mathrm{I}+[\mathrm{K} \mathrm{V}] \lambda 4121$ & 0.187 & $0.64 \pm 0.01$ & $0.91 \pm 0.02$ \\
\hline He I $\lambda 4144$ & 0.182 & $0.730 \pm 0.006$ & $1.021 \pm 0.008$ \\
\hline [K v] $\lambda 4163$ & 0.178 & $0.47 \pm 0.01$ & $0.65 \pm 0.02$ \\
\hline He I + O II $\lambda 4169$ & 0.177 & $0.060 \pm 0.002$ & $0.084 \pm 0.004$ \\
\hline Не I $\lambda 4200$ & 0.170 & $1.14 \pm 0.02$ & $1.55 \pm 0.03$ \\
\hline N II + Fe V $\lambda 4228$ & 0.165 & $0.73 \pm 0.02$ & $0.99 \pm 0.02$ \\
\hline C II $\lambda 4267$ & 0.157 & $0.45 \pm 0.01$ & $0.60 \pm 0.01$ \\
\hline O II $\lambda 4317$ & 0.147 & $0.697 \pm 0.006$ & $0.914 \pm 0.008$ \\
\hline $\mathrm{H} \gamma \lambda 4340$ & 0.143 & $35.97 \pm 0.02$ & $46.79 \pm 0.04$ \\
\hline [O III $] \lambda 4363$ & 0.138 & $28.34 \pm 0.02$ & $36.56 \pm 0.03$ \\
\hline $\mathrm{Ne}$ II + N III $\lambda 4379$ & 0.136 & $0.49 \pm 0.01$ & $0.62 \pm 0.01$ \\
\hline He I $\lambda 4388$ & 0.134 & $0.84 \pm 0.01$ & $1.08 \pm 0.01$ \\
\hline O II $\lambda 4415$ & 0.127 & $0.096 \pm 0.004$ & $0.121 \pm 0.006$ \\
\hline Не I $\lambda 4438$ & 0.120 & $0.091 \pm 0.003$ & $0.114 \pm 0.004$ \\
\hline He I $\lambda 4471$ & 0.110 & $5.63 \pm 0.01$ & $6.89 \pm 0.02$ \\
\hline He II $\lambda 4541$ & 0.089 & $2.07 \pm 0.01$ & $2.43 \pm 0.01$ \\
\hline $\operatorname{Mg} \mathrm{I}] \lambda 4571$ & 0.080 & $0.47 \pm 0.01$ & $0.55 \pm 0.01$ \\
\hline [Fe III] + N II $\lambda 4606$ & 0.070 & $0.902 \pm 0.008$ & $1.026 \pm 0.009$ \\
\hline$[\mathrm{Ar} \mathrm{v}] \lambda 4625$ & 0.064 & $0.320 \pm 0.009$ & $0.36 \pm 0.01$ \\
\hline N III $\lambda 4634$ & 0.062 & $2.099 \pm 0.009$ & $2.35 \pm 0.01$ \\
\hline N III + O II $\lambda 4641$ & 0.060 & $4.433 \pm 0.009$ & $4.95 \pm 0.01$ \\
\hline$[\mathrm{Fe}$ III $]+$ C IV $\lambda 4658$ & 0.055 & $0.957 \pm 0.007$ & $1.059 \pm 0.008$ \\
\hline He II $\lambda 4686$ & 0.047 & $57.16 \pm 0.02$ & $62.36 \pm 0.02$ \\
\hline Fe III or O II $\lambda 4686$ & 0.043 & $0.43 \pm 0.02$ & $0.47 \pm 0.02$ \\
\hline [Ar IV] + He I $\lambda 4712$ & 0.040 & $13.28 \pm 0.02$ & $14.29 \pm 0.02$ \\
\hline$[\mathrm{Ne}$ IV $] \lambda 4725$ & 0.036 & $2.93 \pm 0.01$ & $3.13 \pm 0.01$ \\
\hline [Ar IV] $\lambda 4740$ & 0.032 & $15.40 \pm 0.02$ & $16.34 \pm 0.02$ \\
\hline $\mathrm{H} \beta \lambda 4861$ & 0.000 & $100.00 \pm 0.04$ & $100.00 \pm 0.04$ \\
\hline [Fe III] $\lambda 4881$ & -0.005 & $0.439 \pm 0.007$ & $0.435 \pm 0.007$ \\
\hline [Fe VII] $\lambda 4893$ & -0.008 & $0.535 \pm 0.004$ & $0.527 \pm 0.004$ \\
\hline He I $\lambda 4922$ & -0.015 & $1.976 \pm 0.005$ & $1.920 \pm 0.005$ \\
\hline [O III] $\lambda 4932$ & -0.018 & $0.636 \pm 0.005$ & $0.616 \pm 0.005$ \\
\hline
\end{tabular}

Table A.1. continued.

\begin{tabular}{|c|c|c|c|}
\hline Line & $f(\lambda)$ & $F(\lambda)$ & $I(\lambda)$ \\
\hline [Fe VII] $\lambda 4943$ & -0.021 & $0.544 \pm 0.005$ & $0.523 \pm 0.005$ \\
\hline [O III] $\lambda 4959$ & -0.025 & $466.9 \pm 0.3$ & $446.1 \pm 0.3$ \\
\hline$[\mathrm{Fe}$ VI] $\lambda 4972$ & -0.028 & $0.149 \pm 0.003$ & $0.142 \pm 0.003$ \\
\hline$[\mathrm{Fe}$ VII $] \lambda 4989$ & -0.032 & $0.351 \pm 0.008$ & $0.331 \pm 0.008$ \\
\hline$[\mathrm{O}$ III $] \lambda 5007$ & -0.037 & $1433.4 \pm 0.6$ & $1339.9 \pm 0.6$ \\
\hline Si II] $\lambda 5040$ & -0.045 & $1.58 \pm 0.03$ & $1.45 \pm 0.02$ \\
\hline He I $\lambda 5048$ & -0.047 & $0.31 \pm 0.02$ & $0.29 \pm 0.02$ \\
\hline $\mathrm{Si}$ II] $\lambda 5055$ & -0.049 & $0.86 \pm 0.02$ & $0.79 \pm 0.02$ \\
\hline$[\mathrm{Fe} \mathrm{VI}] \lambda 5145$ & -0.070 & $0.66 \pm 0.02$ & $0.58 \pm 0.02$ \\
\hline$[\mathrm{Fe}$ VII] $\lambda 5159$ & -0.073 & $0.78 \pm 0.02$ & $0.68 \pm 0.02$ \\
\hline$[\mathrm{Fe} \mathrm{VI}] \lambda 5176$ & -0.077 & $0.57 \pm 0.01$ & $0.50 \pm 0.01$ \\
\hline$[\mathrm{N} \mathrm{I}] \lambda 5200$ & -0.082 & $30.33 \pm 0.02$ & $26.09 \pm 0.02$ \\
\hline$[\mathrm{Fe}$ II $] \lambda 5261$ & -0.096 & $0.266 \pm 0.006$ & $0.223 \pm 0.005$ \\
\hline$[\mathrm{Fe}$ III $] \lambda 5270$ & -0.098 & $0.296 \pm 0.005$ & $0.247 \pm 0.004$ \\
\hline [Fe VII] $\lambda 5276$ & -0.099 & $0.247 \pm 0.007$ & $0.206 \pm 0.006$ \\
\hline$[\mathrm{O}$ VI] $\lambda 5291$ & -0.102 & $0.453 \pm 0.003$ & $0.375 \pm 0.002$ \\
\hline$[\mathrm{Ca} \mathrm{V}] \lambda 5309$ & -0.106 & $0.655 \pm 0.005$ & $0.538 \pm 0.004$ \\
\hline [Cl IV] $\lambda 5323$ & -0.109 & $0.327 \pm 0.003$ & $0.267 \pm 0.003$ \\
\hline$[\mathrm{Fe} \mathrm{VI}] \lambda 5335$ & -0.112 & $0.470 \pm 0.005$ & $0.383 \pm 0.004$ \\
\hline He II $\lambda 5412$ & -0.128 & $7.32 \pm 0.01$ & $5.785 \pm 0.008$ \\
\hline$[\mathrm{Fe} \mathrm{VI}] \lambda 5424$ & -0.131 & $0.640 \pm 0.007$ & $0.503 \pm 0.005$ \\
\hline$[\mathrm{Ca} \mathrm{VI}] \lambda 5461$ & -0.138 & $0.61 \pm 0.01$ & $0.47 \pm 0.01$ \\
\hline [Cl III] $\lambda 5517$ & -0.149 & $1.366 \pm 0.007$ & $1.038 \pm 0.005$ \\
\hline [Cl III $] \lambda 5537$ & -0.153 & $1.603 \pm 0.009$ & $1.209 \pm 0.007$ \\
\hline [O I] $\lambda 5577$ & -0.160 & $0.230 \pm 0.002$ & $0.171 \pm 0.001$ \\
\hline O III $\lambda 5592$ & -0.163 & $0.271 \pm 0.007$ & $0.201 \pm 0.005$ \\
\hline K VI $\lambda 5602$ & -0.165 & $0.311 \pm 0.006$ & $0.230 \pm 0.005$ \\
\hline$[\mathrm{Fe}$ VI] $\lambda 5631$ & -0.170 & $0.444 \pm 0.004$ & $0.325 \pm 0.003$ \\
\hline $\mathrm{N}$ II] $\lambda 5667$ & -0.176 & $0.325 \pm 0.006$ & $0.235 \pm 0.004$ \\
\hline $\mathrm{N}$ II $+[\mathrm{Fe}$ VI $] \lambda 5679$ & -0.178 & $0.531 \pm 0.004$ & $0.383 \pm 0.002$ \\
\hline$[\mathrm{Fe}$ VII $] \lambda 5720$ & -0.185 & $1.252 \pm 0.007$ & $0.890 \pm 0.005$ \\
\hline$[\mathrm{N}$ II $] \lambda 5755$ & -0.191 & $32.74 \pm 0.02$ & $23.05 \pm 0.02$ \\
\hline He I $\lambda 5876$ & -0.210 & $32.96 \pm 0.04$ & $22.37 \pm 0.03$ \\
\hline He II + Sky $\lambda 5914$ & -0.217 & $0.46 \pm 0.02$ & $0.31 \pm 0.01$ \\
\hline He II $\lambda 5932$ & -0.219 & $0.41 \pm 0.01$ & $0.239 \pm 0.007$ \\
\hline He II $\lambda 5953$ & -0.223 & $0.275 \pm 0.005$ & $0.159 \pm 0.003$ \\
\hline He II $\lambda 5977$ & -0.226 & $0.647 \pm 0.004$ & $0.427 \pm 0.003$ \\
\hline He II $\lambda 6004$ & -0.230 & $0.514 \pm 0.002$ & $0.336 \pm 0.002$ \\
\hline He II $\lambda 6037$ & -0.235 & $0.645 \pm 0.005$ & $0.418 \pm 0.003$ \\
\hline He II $\lambda 6074$ & -0.241 & $0.631 \pm 0.004$ & $0.405 \pm 0.002$ \\
\hline Fe VII $\lambda 6086$ & -0.243 & $2.202 \pm 0.008$ & $1.408 \pm 0.005$ \\
\hline K IV $\lambda 6102$ & -0.245 & $1.510 \pm 0.005$ & $0.961 \pm 0.003$ \\
\hline He II $\lambda 6118$ & -0.247 & $0.679 \pm 0.003$ & $0.430 \pm 0.002$ \\
\hline He II $\lambda 6171$ & -0.255 & $0.798 \pm 0.005$ & $0.426 \pm 0.003$ \\
\hline He II $\lambda 6234$ & -0.264 & $1.79 \pm 0.02$ & $0.934 \pm 0.009$ \\
\hline [O I] $\lambda 6300$ & -0.274 & $54.0 \pm 0.1$ & $27.48 \pm 0.09$ \\
\hline [S III] $\lambda 6312$ & -0.275 & $11.24 \pm 0.03$ & $5.71 \pm 0.02$ \\
\hline Si II $\lambda 6347$ & -0.280 & $0.71 \pm 0.02$ & $0.35 \pm 0.01$ \\
\hline$[\mathrm{O} \mathrm{I}] \lambda 6363$ & -0.282 & $19.04 \pm 0.04$ & $9.50 \pm 0.03$ \\
\hline [Mn v] $\lambda 6394$ & -0.286 & $0.328 \pm 0.003$ & $0.162 \pm 0.002$ \\
\hline He II $\lambda 6406$ & -0.288 & $0.745 \pm 0.005$ & $0.367 \pm 0.003$ \\
\hline [Ar v] $\lambda 6435$ & -0.292 & $8.22 \pm 0.02$ & $4.01 \pm 0.01$ \\
\hline $\mathrm{He}$ II $+[\mathrm{N}$ II $] \lambda 6527$ & -0.304 & $0.99 \pm 0.08$ & $0.47 \pm 0.04$ \\
\hline$[\mathrm{N}$ II $] \lambda 6548$ & -0.307 & $528 \pm 1$ & $248.6 \pm 0.7$ \\
\hline $\mathrm{H} \alpha \lambda 6563$ & -0.309 & $610 \pm 1$ & $285.9 \pm 0.8$ \\
\hline$[\mathrm{N}$ II] $\lambda 6584$ & -0.311 & $1636 \pm 3$ & $761 \pm 2$ \\
\hline He I $\lambda 6678$ & -0.323 & $11.59 \pm 0.03$ & $5.24 \pm 0.02$ \\
\hline [S II] $\lambda 6717$ & -0.328 & $55.5 \pm 0.2$ & $24.81 \pm 0.09$ \\
\hline$[\mathrm{S} \mathrm{II}] \lambda 6731$ & -0.330 & $81.5 \pm 0.2$ & $36.28 \pm 0.11$ \\
\hline$[\mathrm{K}$ IV] $\lambda 6795$ & -0.337 & $0.824 \pm 0.008$ & $0.360 \pm 0.004$ \\
\hline$[\mathrm{Mn}$ II $] \lambda 6850 ?$ & -0.344 & $1.23 \pm 0.04$ & $0.53 \pm 0.02$ \\
\hline
\end{tabular}


A. B. Rauber et al.: Spectroscopic mapping of the planetary nebula NGC 6302

Table A.1. continued.

\begin{tabular}{llll}
\hline \hline Line & $f(\lambda)$ & $F(\lambda)$ & $I(\lambda)$ \\
\hline He II $\lambda 6891$ & -0.349 & $2.21 \pm 0.05$ & $0.94 \pm 0.02$ \\
[Ar V] $\lambda 7005$ & -0.362 & $21.76 \pm 0.05$ & $8.94 \pm 0.03$ \\
He I $\lambda 7065$ & -0.371 & $22.57 \pm 0.05$ & $9.08 \pm 0.03$ \\
[Ar III] $\lambda 7136$ & -0.381 & $77.0 \pm 0.1$ & $30.21 \pm 0.09$ \\
[Fe II] $\lambda 7155$ & -0.383 & $0.51 \pm 0.02$ & $0.199 \pm 0.007$ \\
[Ar IV] $\lambda 7171$ & -0.386 & $4.81 \pm 0.02$ & $1.866 \pm 0.009$ \\
[Ar IV] $\lambda 7263$ & -0.398 & $2.73 \pm 0.01$ & $1.026 \pm 0.006$ \\
He I $\lambda 7298$ & -0.403 & $0.39 \pm 0.04$ & $0.14 \pm 0.02$ \\
[O II] $\lambda 7319$ & -0.405 & $26.06 \pm 0.05$ & $9.62 \pm 0.03$ \\
[O II] $\lambda 7330$ & -0.407 & $20.23 \pm 0.08$ & $7.45 \pm 0.04$ \\
[Fe II] $\lambda 7453$ & -0.423 & $1.70 \pm 0.01$ & $0.603 \pm 0.004$ \\
He II + C IV $\lambda 7593$ & -0.440 & $5.15 \pm 0.06$ & $1.75 \pm 0.02$ \\
C IV] $\lambda 7737 ?$ & -0.457 & $1.81 \pm 0.03$ & $0.59 \pm 0.01$ \\
[Ar III] $\lambda 7751$ & -0.459 & $23.31 \pm 0.07$ & $7.54 \pm 0.03$ \\
[Cl IV] $\lambda 8046$ & -0.493 & $5.03 \pm 0.02$ & $1.501 \pm 0.007$ \\
He II $\lambda 8237$ & -0.513 & $4.78 \pm 0.02$ & $1.357 \pm 0.008$ \\
H I P15 $\lambda 8545$ & -0.544 & $2.728 \pm 0.009$ & $0.717 \pm 0.003$ \\
[Cl II] $\lambda 8578$ & -0.547 & $2.27 \pm 0.01$ & $0.592 \pm 0.004$ \\
[Fe II] $\lambda 8617$ & -0.551 & $1.40 \pm 0.05$ & $0.36 \pm 0.01$ \\
H I P14 $\lambda 8665$ & -0.555 & $3.47 \pm 0.09$ & $0.89 \pm 0.02$ \\
N I $\lambda 8680$ & -0.557 & $1.84 \pm 0.02$ & $0.468 \pm 0.006$ \\
He II $\lambda 8701$ & -0.559 & $0.34 \pm 0.02$ & $0.087 \pm 0.004$ \\
[C I] $\lambda 8727$ & -0.561 & $2.17 \pm 0.01$ & $0.547 \pm 0.004$ \\
HI P12 $\lambda 8750$ & -0.563 & $4.26 \pm 0.02$ & $1.067 \pm 0.006$ \\
\hline
\end{tabular}

